\title{
Effect of Cumulative Surface on Pore Development in Chalk
}

\author{
Yang, Y.; Hakim, S. S.; Bruns, S.; Uesugi, K.; Stipp, S. L. S.; Sørensen, H. O.
}

Published in:

Water Resources Research

Link to article, DOI:

10.1029/2018WR023756

Publication date:

2019

Document Version

Peer reviewed version

Link back to DTU Orbit

Citation (APA):

Yang, Y., Hakim, S. S., Bruns, S., Uesugi, K., Stipp, S. L. S., \& Sørensen, H. O. (2019). Effect of Cumulative Surface on Pore Development in Chalk. Water Resources Research, 55(6), 4801-4819.

https://doi.org/10.1029/2018WR023756

\section{General rights}

Copyright and moral rights for the publications made accessible in the public portal are retained by the authors and/or other copyright owners and it is a condition of accessing publications that users recognise and abide by the legal requirements associated with these rights.

- Users may download and print one copy of any publication from the public portal for the purpose of private study or research.

- You may not further distribute the material or use it for any profit-making activity or commercial gain

- You may freely distribute the URL identifying the publication in the public portal

If you believe that this document breaches copyright please contact us providing details, and we will remove access to the work immediately and investigate your claim 
Yang Yi (Orcid ID: 0000-0001-8270-3006)

Hakim Sepideh Sadat (Orcid ID: 0000-0001-6486-4551)

Bruns S. (Orcid ID: 0000-0003-1945-0549)

Uesugi Kentaro (Orcid ID: 0000-0003-2579-513X)

Sørensen Henning Osholm (Orcid ID: 0000-0002-7004-547X)

\title{
Effect of Cumulative Surface on Pore Development in Chalk
}

\author{
Y. Yang, ${ }^{1 \dagger}$ S. S. Hakim, ${ }^{1 \ddagger}$ S. Bruns, ${ }^{1 \ddagger}$ K. Uesugi, ${ }^{2}$ S. L. S. Stipp, ${ }^{1 \ddagger}$ and H. O. Sørensen ${ }^{1 \ddagger}$
}

${ }^{1}$ Nano-Science Center, Department of Chemistry, University of Copenhagen, Universitetsparken 5, DK-2100 Copenhagen, Denmark

${ }^{2}$ Japan Synchrotron Radiation Research Institute / SPring-8, Sayo, Hyogo 679-5198, Japan

${ }^{\dagger}$ PRESENT ADDRESS: Department of Chemistry, Technical University of Denmark, KEMITORVET, DK-2800 Kgs. Lyngby, Denmark

* PRESENT ADDRESS: Department of Physics, Technical University of Denmark, FYSIKVEJ, DK-2800 Kgs. Lyngby, Denmark

Corresponding author: Yi Yang (yyan@dtu.dk)

\section{Key Points:}

- Cumulative surface is the surface area that is accessible to reactive fluid along a flow path within the fluid's residence time

- A lower cumulative surface yields a lower reactant conversion and thus farther spreading of reactant in the flow direction

- When pore development stems from the advection-dissolution coupling, streamlines with minimum cumulative surface are favored pathways because they host the farthest reaching dissolution front

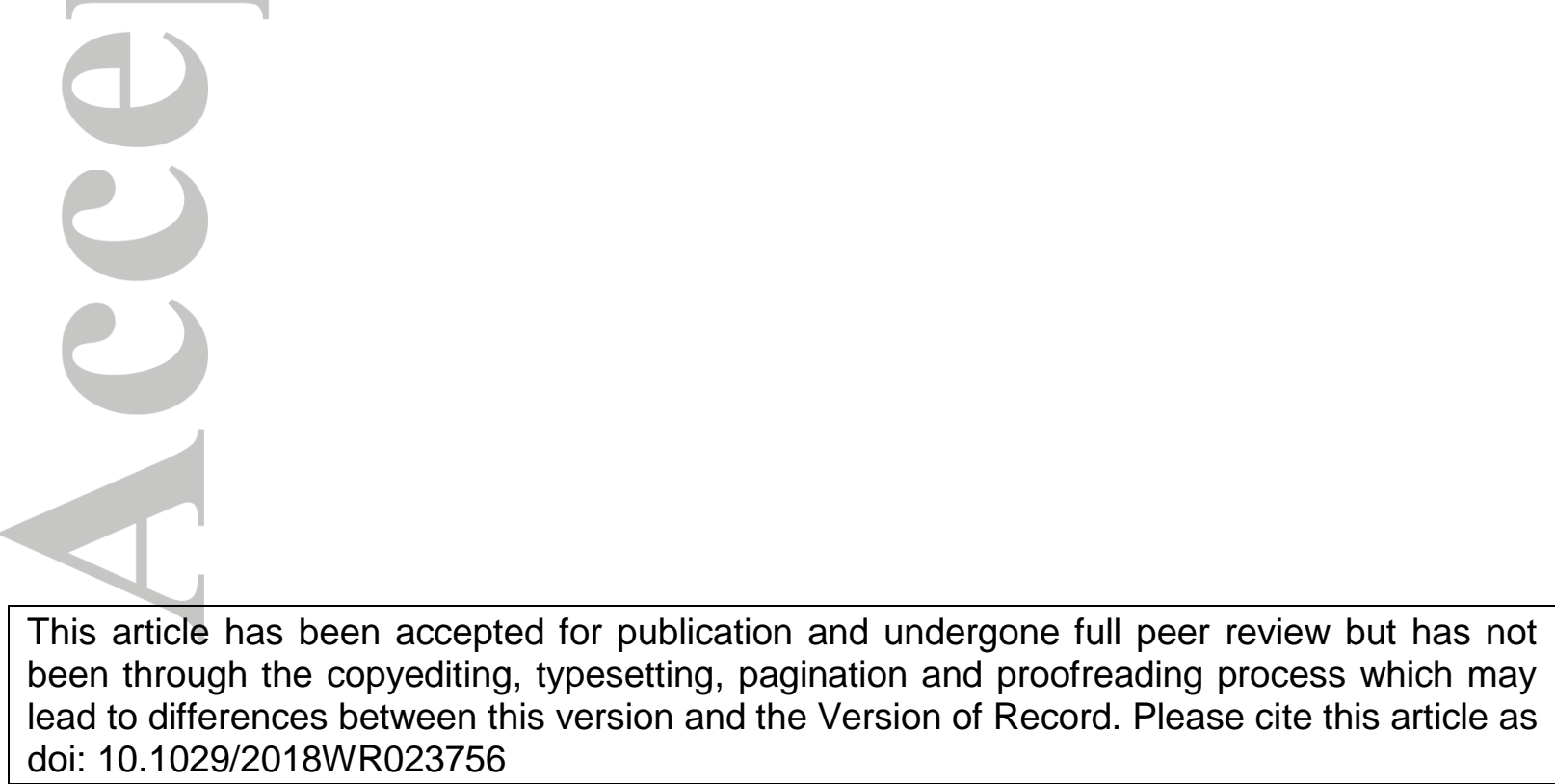

(C) 2019 American Geophysical Union. All rights reserved. 


\begin{abstract}
Pore development in natural porous media, as a result of mineral dissolution in flowing fluid, generates complex microstructures. Although the underlying dynamics of fluid flow and the kinetics of the dissolution reactions have been carefully analyzed in many scenarios, it remains interesting to ask if the preferentially developed flow paths share certain general petrophysical properties. Here we combine in situ X-ray imaging with network modeling to study pore development in chalk driven by acidic fluid flow under ambient condition. We show that the trajectory of a growing pore correlates with the flow path that minimizes cumulative surface - the overall surface area available to fluid within the residence time calculated along streamlines. This correlation is not a coincidence because cumulative surface determines conversion of reactant and thus defines the position of dissolution front. Model simulations show that, as fluid channelizes, the growth of the leading pore in the flow direction is guided by migration of the most far-reaching dissolution front, even in an everchanging flow field. In addition, we present a complete tomographic time series of microstructure erosion and show a good accord between the in situ observation and the model simulation, Our results suggest that the microscopic pore development is a deterministic process while being sensitive to stochastic perturbations to the migrating dissolution front.
\end{abstract}

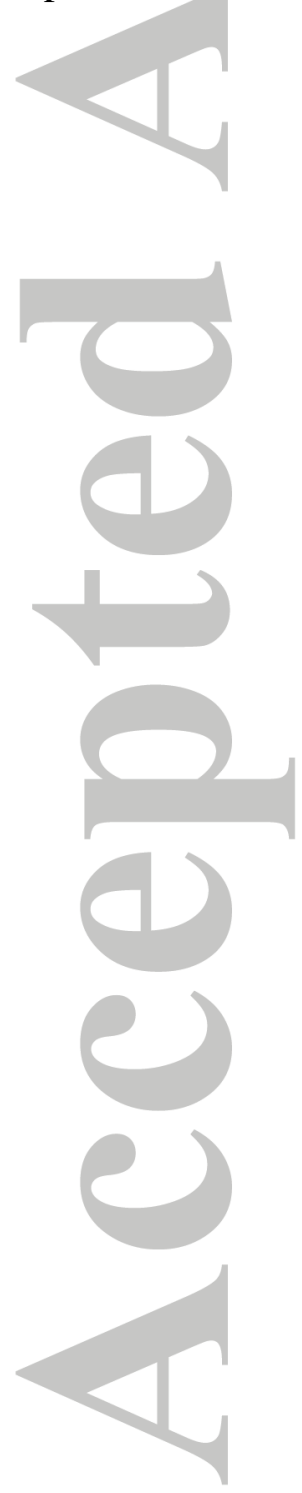




\section{Introduction}

The microscopic development of self-organized flow paths in porous media is ubiquitous in nature (Ortoleva, 1994). Pores compete and develop in parallel when there is positive feedback between fluid flow and mineral dissolution (Szymczak \& Ladd, 2009; Budek \& Szymczak, 2012; Szymczak \& Ladd, 2014). When a reactive fluid flows through a dissolving porous medium, it preferentially erodes the regions it has previously etched (Hinch $\&$ Bhatt, 1990). This process produces highly conductive channels and leads to a stratified distribution of flow velocity.

Understanding the mechanism of microscopic pore development will benefit several scientific disciplines. For example, in geochemistry, all kinetic measurements of water-rock interactions in porous media face the problem of how to normalize for surface area (Maher et al., 2006; Landrot et al., 2012; Molins et al., 2012; Noiriel et al., 2012). The development of bypassing channels renders a significant portion of materials inaccessible to fresh reactant and thus unavailable for reaction. This non-reacting portion introduces error in the estimate of surface area and the chemical affinity used in rate normalization (Li et al., 2006). In geologic carbon sequestration (GCS) (DePaolo \& Cole, 2013; Fitts \& Peters, 2013; Zhang \& Liu, 2016) and in acid stimulation in oil fields (Hoefner \& Fogler, 1988), injecting erosive fluids generates complex flow pathways. Managing the development of such networks, i.e. controlling the rate of network expansion while regulating the size distribution of the growing channels, is essential for properly distributing $\mathrm{CO}_{2}$ into porous formations (Luquot \& Gouze, 2009; Abdoulghafour et al., 2013; Deng et al., 2013; Ellis et al., 2013; Deng et al., 2015; Noiriel, 2015; Yang et al., 2017) and for effectively recovering hydrocarbons from reservoirs (Hoefner \& Fogler, 1988; Rege \& Fogler, 1989; Fredd \& Fogler, 1998).

Identifying the controlling factors for microscopic pore development in natural porous media is an active and fruitful research area, although our current understanding of the underlying mechanisms is occasionally impeded by the controversial tendency of parameterizing a dynamic process using a few stationary, macroscopic parameters. The problem has been formulated in the context of reactive infiltration instability, where a migrating dissolution front is perturbed and morphologically distorted by local variations in transport properties (Chadam et al., 1986; Ortoleva et al., 1987; Szymczak \& Ladd, 2012; Zhao et al., 2012; Zhao et al., 2015). The instability is determined by the strength of the coupling among three parameters that vary spatially: velocity, permeability and reactant concentration. The flow field depends on permeability variations, while the latter further depends on distribution of reactants that dissolve/precipitate solid, as well as on mineralogical composition and heterogeneity. The reactivity field relies on fluid flow for reactant transport, thus closing the feedback loop. If any of these dependencies vanishes, the loop breaks and channelized microstructure does not form. For example, if there is no imposed flow, the dissolution front remains diffusive and planar (Daccord, Lenormand, et al., 1993; Daccord, Lietard, et al., 1993; Fredd \& Fogler, 1998; Fredd \& Fogler, 1999). Similarly, a zeroth order dissolution reaction does not channelize fluid because its rate is independent of reactant concentration. In two phase flow in GCS, this coupling might also break if gaseous $\mathrm{CO}_{2}$, which has a lower reactivity than the acidified brine, preferentially travels toward "wormhole seeds" (Holger \& Sjaam, 2015). This character of fluid distribution reverses the positive feedback in that the more permeable flowpath becomes less reactive. As a result, the reaction front is more stable in the presence of non-aqueous $\mathrm{CO}_{2}$, and a compact dissolution pattern is favored over wormholing.

Chalk is an important bedding rock in groundwater aquifers and oil and gas reservoirs in Northwest Europe. Pore development stemming from preferential erosion of calcite in 
flowing fluid is a ubiquitous and highly reproducible phenomenon in chalk (Fig. S1). Our group previously studied the importance of dissolution front migration on shaping pore development in chalk (Yang, Bruns, Rogowska, et al., 2018; Yang, Hakim, et al., 2018). However, it remains unclear whether static petrophysical parameters of chalk can be used to understand the dynamics of dissolution front migration and therefore local pore development. The key is to combine permeability and surface area into one parameter in the context of medium dissolution. Permeability determines the residence time distribution of reactant while within this time the access to surface area defines the actual conversion from reactant to product. In a recent paper Wen and Li reformulated the problem of mineral dissolution in porous media and proposed that the effective rate could be decomposed into three components (Wen \& $\mathrm{Li}, 2018$ ). The first is a constant that reflects the laboratory rate and the static geometric surface area. The second reflects the exponential decay of effective surface area as fluid approaches equilibrium. This term is analogous to one that describes fluid reactivity decay in an ideal plug flow reactor. It underlines the inherent connection between reactive surface area and the Gibbs free energy dependence of mineral dissolution. This term also provides a way to estimate the size of the reactive subvolume. The third component quantifies the impact of medium heterogeneities. When the reactive subvolume is small compared to the whole domain, heterogeneities are of less concern because they mostly fall outside this subvolume. In contrast, when the reactive subvolume is large (e.g. this corresponds to a faster flowrate or a smaller/shorter domain), the heterogeneities within affects reactant consumption significantly. It is worth mentioning that in this formulation the specific surface area is considered uniform in the flow direction and that the heterogeneities are those related to fluid flow and advective mass transfer.

In this study, we define Cumulative Surface (CS) as the amount of surface area to which fluid has access within the residence time. This concept was first coined to quantify the reactive subvolume of porous media (Yang et al., 2017; Yang, Bruns, Rogowska, et al., 2018). Here we further argue that CS, by integrating the effects of residence time, reaction rate and initial pore heterogeneity into one quantity, also helps identify the trajectory of localized pore development. This speculation stems from the characteristic of the positive feedback loop, which amplifies heterogeneity over time. This amplifying mechanism assures that the leading flow path - one that has the farthest reaching reaction front - leads throughout a process dominated by the positive feedback. We combine model simulation with in situ X-ray imaging to show that the path of the dominant pore correlates with the flow path with the most far reaching reactive fluid. Numerical simulations are run using both static high resolution nanotomographic data (nanoCT) and the in situ microtomographic data

$(\mu \mathrm{CT})$. In addition, a full 3D, in situ imaging time series collected continuously over 21 hours is reported and analysed. Our result serves as the first step in describing the self-organization of flow networks as a population balancing problem: a problem of quantifying the dynamic number of channels. This number is initially determined by the abundance of heterogeneities as pore seeds but ultimately limited by solid availability as a microstructure evolves.

\section{Materials and Methods}

2.1 In situ microtomographic $(\mu \mathrm{CT})$ of chalk dissolution processes

Chalk samples were obtained from the Rørdal Quarry (Maastrichtian age) near Aalborg, Denmark. The samples were predominantly $\mathrm{CaCO}_{3}$ coccoliths and skeletal debris. The silica content of the sample was less than $4 \%$ (Fabricius, 2007). The samples were machined into cylinders of $0.9 \mathrm{~mm}$ diameter and cut to $2 \mathrm{~mm}$ long using a razor blade. This dimension ratio was chosen for practicality: within the machining precision, this ratio 
allowed us to construct a robust fluid cell that hosts the sample, the wrapping polymer and a thin layer of external aluminum shell in the imaging window (in the radial direction) during full cell rotation. Each cylinder was inserted into a heat shrinking tube that was fixed, by heating and with epoxy glue, to two stainless steel needles at each end. Both needles were 3 $\mathrm{cm}$ long and $500 \mu \mathrm{m}$ in diameter. This assembly (sample, shrinking tube and needles) was then loaded into a fluid cell that resembled a miniature of Hassler core holder. The cell had two pairs of inlets and outlets for sample confining and solution percolation, respectively. After mounting the sample vertically in the central aluminum tube ( $2.5 \mathrm{~mm}$ OD), the confining pressure was increased slowly to 10 bar by pumping deionized water into the shell side of the cell (water remained outside the wrapping polymer and did not contact the sample). This pressure held the sample still and ensured that the sleeve was tight during fluid percolation. The shell side was then sealed and the percolating inlet and outlet were opened to allow fluid perfusion in the axial direction inside the wrapping polymer. We used ultrapure (MilliQ) water equilibrated with 1 bar $\mathrm{CO}_{2}$ under ambient temperature $\left(25^{\circ} \mathrm{C}\right)$, which led to an injection $\mathrm{pH}$ of 3.9. The $\mathrm{CO}_{2}$ gas was bubbled constantly into the reservoir of MilliQ (500 $\mathrm{ml}$ ) throughout the experiment. An SSI series II HPLC pump (Scientific Systems, Inc.) was used to maintain a constant flow rate of $0.01 \mathrm{~mL} / \mathrm{min}$. The pressure drop across the cylindrical sample was not specified. The outlet of the fluid cell was connected to an effluent collector that operated under ambient pressure, i.e. the downstream pressure was expected to be $\sim 1$ bar. The upstream pressure varied throughout the experiment as the sample permeability decreased and was not monitored. Although $\mathrm{CO}_{2}$ degassing stemming from hydraulic pressure drop was assumed during signal processing for generality, it was not visually observed in the images nor was there discernible effect on the signal intensity in the histograms.

A time series of $\mu \mathrm{CT}$ 3D images collected in situ allows direct observation of preferential flow path formation in real time and thus provides firsthand information on the dynamics of microstructural evolution. The requirements for in situ imaging are typically more stringent than those for ex situ because of practicalities associated with the rotating, semi-open sample environment with continuous fluid flow (Yang, Bruns, Rogowska, et al., 2018; Yang, Hakim, et al., 2018). The time resolved microtomography experiments were conducted at the BL20XU medical beamline at SPring-8, Japan (Uesugi et al., 2012). The dissolution process was monitored by recording - continuously over 21 hours - tomography datasets. For every data set 1,800 projections were recorded while rotating the sample $180^{\circ}$ with an exposure time of 1 second per projection using X-ray radiation of $28 \mathrm{keV}$. 30 dark field images were recorded before, and 30 bright field images were taken before and after each rotation. This resulted in time series with a time resolution of $\sim 40 \mathrm{~min}$. The projections of $2048 \times 2048$ pixels were acquired with a $20 \times$ objective lens, yielding an effective pixel size of $248 \mathrm{~nm}$ and a field of view of $0.5 \times 0.5 \mathrm{~mm}^{2}$.

After collection, the in situ data were both visually inspected and used in numerical simulation. The 3D reconstructions of each tomographic dataset were performed using the GridRec reconstruction algorithm implemented with TomoPy (Gürsory et al., 2014). A uniform greyscale distribution in the reconstructed image stacks was assured by mapping $99.98 \%$ of the dynamic range in every reconstructed 2D slice to a 0 to 1 interval in a 32-bit greyscale image (Bruns et al., 2017a). All reconstructions were then cropped because the flow cell incorporates four stabilizing stainless steel rods that shade some of the projections and thereby cause streaking artefacts at the edges of the reconstruction. The cropped reconstructions were further processed by manually estimating the noise level from homogeneous regions of void and material phases and applying four iterations of 3D iterative nonlocal means denoising (Bruns et al., 2017b, 2017a). Alignment of the 4D time series was 
performed after denoising with in-house digital volume correlation software using Pearson's correlation coefficient as a quality metric.

Degassing of $\mathrm{CO}_{2}$ may occur, causing the fluid density to vary from inlet to outlet, i.e. local voxel porosity can only be approximated. This was done by fitting a Gaussian mixture model to the greyscale histogram and assigning a soft segmentation: high porosity phase, low porosity phase and nonporous (solid) phase. Expected mean greyvalues for the void and solid phase were determined by tracking voxels that did not change intensity over time. The expected porosity for the mixed phases was then determined by linear interpolation between void and material phase. For the microtomography reconstructions, the fuzzy phase assignment was further refined by optimizing a Markov random field model, where the interface potentials between the four phases were defined by their difference in expected material content. Ultimately, a continuous expression for local voxel porosity was calculated as an average, weighted by the local phase membership probabilities. See also Bruns et al. (2017b) for details.

\subsection{Greyscale modeling and numerical simulation}

Greyscale modeling uses the greyvalues of voxels in the tomographic reconstruction to parameterize model elements. This approach does not segment tomographic data and thus preserves high geometric fidelity in model simulation. The porous medium is considered continuum on the voxel scale, and the tomographic image is used to populate the value of porosity, from which the other parameters are derived. A similar strategy has been adopted in the micro-continuum approach (Steefel et al., 2015, Soulaine et al., 2017). We used two types of tomographic data in model simulation: the in situ $\mu \mathrm{CT}$ data (Section 2.1) and an ex situ nanotomography (nanoCT) dataset from previous study. The nanoCT data were collected on Hod chalk with a field of view of $0.25 \mathrm{~mm}^{2}$ (Müter et al., 2014). We used the nanoCT data for numerical analysis and theoretical prediction (Section 3.1) and used $\mu \mathrm{CT}$ data for the validation of experimental results (Section 3.3). The reason for this choice is that the simulations based on the $\mu \mathrm{CT}$ data allowed us to directly compare numerical results with experimental observations. However, the stringent in situ recording conditions were not ideal for optimization of imaging quality, and the surface area thus attained was less accurate. In contrast, the nanoCT data, collected on dry chalk samples, had higher imaging resolution (25 $\mathrm{nm} /$ voxel) and was most amenable to the signal processing techniques developed in house (Bruns et al., 2017b, 2017a), i.e. it was a high quality digital geometry model for numerical simulation.

Greyscale modeling is desirable for chalk dissolution. Voxel represents a hard limit of structural characterization using tomography. Chalk is a very fine-grained rock and its structural information exists beyond characterization resolution of X-ray imaging. Lin et al. used KI solution to enhance the contrast between different minerals and retrieved sub-voxel porosity information using a multi-Gaussian mixture model (Lin et al., 2016). A similar approach is adopted in this study. It remains an open question how accurately the sub-voxel structural information can be derived using tomographic techniques. However, there is a consensus that both porosity and surface area exist within voxels - and the apparent scale separation edging on the voxel size does not reflect the lack of such consensus but the ultimate limit of the characterization technique. Modeling processes on a scale comparable to or smaller than a voxel must rely, to a large extent, on educated guesses. In this study, on the voxel scale $(25 \mathrm{~nm})$, we used $i$ ) Darcy's law to describe fluid flow, ii) a quadratic function to relate permeability with porosity, iii) porosity contrast of neighboring voxels to define surface area and $\boldsymbol{i} v$ ) the mixing factor, $\eta$, to quantify sub-voxel mixing. These are interrelated, consequential assumptions that ought to be justified before we dive into further 
discussions. It is worth mentioning that our goal is not to construct a model that would fully reproduce the physical reality numerically. Instead, we used the assumptions from the perspective of assembling knowledge - i.e. as modules in a conceptual model that describes a phenomenon that stemmed from multi-process coupling. The governing equations in each module can always be replaced to reflect new understanding of the subject - or simply to improve the fidelity of simulation at the expense of computational power. The first three assumptions are discussed below along with the model presentation. The justification of using the mixing factor $\eta$ to quantify local mixing is presented in the SI.

A reactor network model was used to combine the spatial distribution of the voxel porosity with the calcite dissolution rate in an imposed flow field (Yang et al., 2017; Yang, Bruns, Stipp, et al., 2018a). Each voxel was modelled as a mixed flow reactor (MFR, Fig. 1a) and was connected to the six neighboring voxels by plug flow reactors (PFR, Fig. 1b). It is worth emphasizing that the actual governing equations for pressure drop or the permeabilityporosity relationship is unknown on the voxel level. We would nonetheless need to evaluate an exemplary flow field and use its streamlines to demonstrate the concept of cumulative surface. This method is analogous to a pore network extraction in that the physical reality is not recreated numerically for predictive purpose but approximated as a basis for assembling knowledge and to advance discussion. Here we assume that the pressure drop occurs in PFR and follows Darcy's law:

$$
q=R z \cdot l_{n} \varphi^{2} \cdot \Delta p
$$

where the permeability depends on the square of voxel porosity. The dots are multiplication signs that separate groups of quantities for readability (e.g. $R z$ depends on petrophysical properties, $l_{n} \varphi^{2}$ on voxel property, while $q$ and $\Delta p$ are dependent and independent variables, respectively). Please see Table 1 for the description of variables, their dimension and the values of the quantities used in the simulation. The absence of unit in Table 1 indicates that the corresponding entry is dimensionless. These dimensionless quantities are either combinations of quantities whose units cancel out or variables and parameters that are scaled to corresponding reference values.

Here we adopt Darcy's law to calculate an exemplary flow field and use its streamlines to illustrate the concept of cumulative surface. Equation 1 and its parameters (below) would affect the exact shapes of streamlines - their (in)validity does not challenge the existence of streamlines, which would be the basis of the discussion in the next section. We admit the caveats - the validity of the governing equation is limited by the flow region and the number of phases involved. The parameterization of voxel level permeability is also different from, e.g. the widely used Kozeny-Carman equation, and lacks verifiability. Muljadi et al. studied the onset of non-Darcy flow behavior in both artificial and natural porous media. The authors showed that the same macroscopic Reynolds number yields different flow behaviors in heterogeneous media and vice versa. They concluded that the appearance of steady eddies led to an increased contribution of the non-Darcy term in the Darcy-Brinkman equation (Muljadi et al., 2016). Their work also highlights the uncertainty of using a global dimensionless number to describe porous media with internal heterogeneities. In addition, Muljadi et al. confirmed the presence of non-Fickian behavior in several porous media, across six orders of magnitude, by tracking the migration of hydrochloric acid plume using nuclear magnetic resonance (NMR) (Muljadi et al., 2017).

Despite the uncertain physical significance in some scenarios, Equation 1 yields a reasonable exemplary flow field. This is because the equation fully utilizes the microstructural information retrieved by the greyscale X-ray tomography and reflects the 
general understanding that more porous regions are more permeable. One could instead choose to segment the greyscale data and solve the Navier-Stokes equations for the pore space. This explicit approach has a concrete physical basis but sacrifices a tremendous amount of geometric information to attain that basis - the segmentation abruptly divides voxels into two porosity values, either 0 or 1 . The 32-bit 3D geometry is thus reduced to 1bit. For rocks with very fine features - e.g. chalk - it is questionable whether the gain in the physical basis of the governing equations could compensate for the dramatic loss of fidelity in microstructure. Alternatively, Raeini et al. proposed a generalized network extraction method for parameterizing 3D digital models of porous media. The method avoids oversimplification of pore structure by assembling different microscopic topological features hierarchically into a coarse discretization of pore space (Raeini et al., 2017). The various features can be implemented selectively into various types of numerical simulations based on their relevance. For example, the authors reported a study on capillary-dominated two-phase flow in which the results were compared with direct finite-volume two-phase flow simulations and with conventional pore-network model (Raeini et al., 2018). The pore network model is another example that non-first principle governing equations could be used to simulate reactive transport in natural porous materials.

The geometric mean of the voxel porosities associated with the PFR (e.g., Voxel 1 and Voxel 2 in Figure 1b) was used in Equation 1. We consider this homogenization (i.e. the material within each voxel is homogeneous) a reasonable simplification given that the voxel size is very small $(25 \mathrm{~nm}) . R z$ is a dimensionless number that characterizes the dependence of the PFR permeability on the neighboring voxels,

$R z=\left(\frac{P_{r e f} L_{r e f}}{Q_{r e f}}\right) \cdot\left(\frac{a}{\mu}\right)$.

The symbol $a$ (unit: $\mathrm{m}^{2}$, see Table 1 ) is the proportionality between voxel level permeability and porosity, i.e. $k_{\text {voxel }}=a \varphi^{2}$. It lumps the dependence of tortuosity and permeability on porosity into a single, phenomenological coefficient. Assuming an incompressible fluid and continuity, a dataset containing 40 million voxels (200 $\square 200 \square 1000$ ) leads to a system of 159.56 million equations, in which 40 million describe the fluid conservation in the MFRs and the remaining 119.56 million, the pressure drop in the PFRs that interconnect neighboring voxels. The system of equations was solved using the stabilized biconjugate gradient method. The flow field was imposed by assigning the injection of fluid as source terms in the inlet reactors and the effluent removal as sink terms in the outlet reactors. The equivalent free stream fluid velocity was $5 \mu \mathrm{m} / \mathrm{s}$. The speciation of the injection fluid was one that pre-equilibrated with $\mathrm{CO}_{2}\left(1 \mathrm{bar}, 25^{\circ} \mathrm{C}\right)$. Degassing of $\mathrm{CO}_{2}$ during percolation was neglected so fluid viscosity did not vary in space. Once the fluid distribution is obtained, the relation between inlet and outlet concentrations of each PFR is given by (Fogler, 2016)

$$
-C_{0}+\frac{k^{\prime}}{k^{\prime}+k^{I}\left(1-e^{D a \cdot k^{\prime}}\right)} \cdot C=0,
$$

where $D a$ is the Damköhler number for the PFR and is defined as:

$$
D a=H n \cdot(1-\eta) \cdot\left(\frac{l_{n}^{2}}{q}\right) \cdot|\Delta \varphi| .
$$


The dimensionless number, $H n$, represents the sensitivity of the calcite dissolution rate to aqueous calcium concentration when the reaction is far from equilibrium

$$
H n=\left(\frac{L_{r e f}^{2}}{Q_{r e f}}\right) \cdot k_{A}^{0} .
$$

In Equation 4, $\eta$ represents the portion of the voxel volume modelled as an MFR. We used $\eta$ instead of the Péclet number in the greyscale model because of the difficulties in obtaining the apparent diffusivities in voxels with varying porosity. This subject is further discussed in detail in the Supporting Information (SI).

In this study, surface is defined as spatial variation of material density (Yeong \& Torquato, 1998). This definition of surface is a necessary extension to greyscale data and is discussed extensively in a related paper (Yang, Bruns, Rogowska, et al., 2018). The definition is compatible with the conventional definition, where the local porosity changes from 0 to 1 in a binary geometry. Meanwhile, this extended definition also considers a change in X-ray intensity between voxels a variation in material density. Given that chalk is predominantly calcium carbonate, this density change reflects a change in local porosity. If the structures within each voxel can be fully resolved, that two voxels with different porosity implicates the existence of physical surface in between. Therefore, this assumption may lead to an underestimate of surface area when voxels are large, or when the spatial variations in porosity is small, but it significantly improves the accuracy of surface area estimation over methods based on binarization, especially for chalk. More specifically, geometric surface area (GSA) measures surface that physically exists, e.g. the porosity contrast of adjacent voxels, $l_{n}^{2} \cdot|\Delta \varphi|$. This quantity does not rely on the presence of fluid and is the upper limit of surface area available at any instant. Reactive surface area (RSA) is the portion of GSA on which a chemical reaction took place: for each reactor, if the outlet concentration, $C$, differs from the inlet, $C_{0}$, then reactant converted and thus the reactor GSA is also RSA. Both GSA and RSA evolution are tracked in our simulation.

The inlet and outlet concentrations of the MFR differ from those of a PFR because of the different residence time distribution. They can be related using

$$
-\sum_{i} q_{i} C_{0, i}+\frac{1-D a^{\prime} \cdot k^{\prime}}{1-D a^{\prime} \cdot\left(k^{I}+k^{\prime}\right)} \cdot q C=0
$$

where $D a$ ' is the Damköhler number for the MFR and is defined as

$$
D a^{\prime}=H n \cdot \eta \cdot\left(\frac{l_{n}^{2}}{q}\right) \cdot \sum_{i=1}^{6}|\Delta \varphi|_{i} .
$$

The summation represents the contribution of GSA from the 6 neighboring voxels. $k^{I}$ is the dimensionless, apparent, first order rate constant defined as $k^{I}=k_{A 0, a p p}^{I} / k_{A}^{0}$, where $k_{A 0, a p p}^{I}=r_{A} /\left(C_{A, \text { eq }}-C_{A 0}\right) . k^{\prime}$ is the dimensionless sensitivity of the calcite dissolution rate to the calcium concentration and is defined as $k^{\prime}=k_{A 0}^{\prime} / k_{A}^{0}$ (Fig. 1c). Both $k^{I}$ and $k^{\prime}$ are auxiliary variables devised to solve the system of governing equations (Eqs $3 \& 6$ ) iteratively. Using the inlet concentration from the previous iteration as the initial guess, the nonlinear rate law for calcite dissolution was applied repeatedly until the Frobenius norm of the difference between the consecutive concentration fields was less than $10^{-6}$. In the numerical simulations, only the transport of total aqueous calcium was tracked. The $\mathrm{pH}$ and the saturation index of 
calcite in each MFR and PFR were computed by assuming calcite as the only source of calcium in a closed compartment. The speciation calculation was conducted with PHREEQC (Parkhurst \& Appelo, 1999), a computer program for speciation, batch-reaction, onedimensional transport, and inverse geochemical calculations. The rate law of calcite dissolution, determined from the rate law proposed by Pokrovsky et al. (Pokrovsky et al., 2005; Pokrovsky et al., 2009), was used to approximate chalk dissolution rate. After the chemical conversion in each reactor (PFR and MFR) was obtained, the voxel porosity was updated according to mass balancing:

$$
d \varphi=D s \cdot l_{n}^{-3} \cdot \sum_{i=1}^{7} q_{i}\left(C_{0, i}-C_{i}\right)
$$

where the dimensionless number, $D s$, defines the time step:

$$
D s=\left(\frac{Q_{r e f}}{L_{r e f}^{3}}\right) \cdot\left(\frac{M}{\rho}\right) \cdot\left(C_{A, \mathrm{eq}}-C_{A, \mathrm{inj}}\right) \cdot d t .
$$

An auto-adaptive algorithm was used to choose the time step, $d t$, so the overall porosity change of the simulation domain was capped at 0.01 , i.e.

$$
\Delta \varphi=\iiint_{V} \frac{1}{H n \cdot l_{n}^{2}} \cdot q \cdot \frac{C_{0}-C}{C_{0}} \cdot d \mathbf{r} \leq 0.01 .
$$

The reference quantities in the simulation were chosen heuristically as:

$$
\begin{aligned}
L_{r e f} & =\frac{\left(C_{A, e q}-C_{A, i n j}\right) d t}{D s} \cdot\left(\frac{M}{\rho}\right) \cdot\left(\frac{k_{A}^{0}}{H n}\right) \\
P_{r e f} & =R z \cdot\left(\frac{\mu}{a}\right) \cdot \frac{\left(C_{A, e q}-C_{A, i n j}\right) d t}{D s} \cdot\left(\frac{M}{\rho}\right) \cdot\left(\frac{k_{A}^{0}}{H n}\right)^{2} \\
Q_{r e f} & =\left[\frac{\left(C_{A, e q}-C_{A, i n j}\right) d t}{D s} \cdot\left(\frac{M}{\rho}\right)\right]^{2} \cdot\left(\frac{k_{A}^{0}}{H n}\right)^{3}
\end{aligned}
$$

The numerical simulations were performed on workstations equipped with Intel Xeon X5677 3.47 GHz processors and 96.0 GB of RAM. Each discrete time step took 36-40 minutes of computing time. Although this domain size is small, with the chosen flowrate it suffices to demonstrate the formation of preferential flow paths and therefore the development of microscopic pores. The microstructure was eventually broken-through by the reactive fluid without dissolving all solid material. In addition, it is not the objective of the current study to propose a representative elementary volume (REV) for pore structure evolution. This subject is discussed in Menke et al. (Menke et al., 2018). The authors studied the impact of heterogeneity on limestone dissolution on both the core and the pore scales and argued that a REV exists for reaction infiltration instability problems but this REV may be greater than the dissolution front in size. The authors also reported that, perpendicular to the flow, porosity evolves alike on different length scales. This observation is in accord with the understanding that infiltration instability stems from the coupling between advection and dissolution and therefore, diffusive mass transfer in the radial direction serves as a negative feedback and does not generate scale-dependent morphological signatures. Diffusion, however, does alleviate the stochastic nature of medium heterogeneity and affects the exact 
shape of newly developed pores. For example, when macroscopic dimensionless numbers are used as measures of reactive transport condition, there are a range of conditions of Pe and Da that lead to conical wormholing where transverse diffusion is important (Daccord, Lietard, et al., 1993; Fredd \& Fogler, 1998). Here we focus on the effect of fluid residence time and the mineral surface that the fluid has access to within the time frame.

\section{Results and Discussion}

3.1 Reaction front migration and pore growth

Simulated Pore Growth and Surface Area Evolution We simulated the microstructure evolution of a chalk cuboid ( $5 \square 5 \square 25 \mu \mathrm{m}^{3}$, extracted from the nanoCT data) during its dissolution in an imposed flow field (Fig. 2). The simulated growth of pore (empty voxels) was sensitive to local variations in porosity near the pore tip (Fig. 2b, yellow arrows). The tip showed a pronounced tendency to evade less permeable regions as it advanced toward the outlet. This phenomenon is characteristic of dissolution in a flow field, where the migrating reaction front is not stable and deviates significantly from a unidirectional plug flow. The structure evolution caused by mineral dissolution provides a positive feedback between the flow rate and the dissolution reaction. This feedback ensures that the flow path with the farthest reaching reaction front better maintains its reactivity compared with other flow paths over time. The heterogeneities in a porous matrix are enhanced, causing ducts to form and fluids to channel. The result is that the fluid breaks through without fully dissolving all solid. In the simulation, breakthrough occurred between porosity 0.4 and 0.6 (Figure 2c) and was manifested by a sharp decrease in the pressure drop in the flow direction (Yang et al., 2017; Yang, Bruns, Stipp, et al., 2018b).

The evolution of geometric and reactive surface area also showed the characteristics of unstable dissolution front migration (Fig. 2c). Similar trends have previously been observed experimentally and used to interpret a transient recession of dissolution front (Yang, Bruns, Rogowska, et al., 2018). After fluid breakthrough, the dissolution pattern was dominated by the expansion of the major flow channel when a constant fluid flux was imposed, and the GSA decreased as solid material depleted locally. This result should be compared with the work of Menke et al., who argue that channeling is fundamentally different from wormholing (Menke et al., 2016). In the paper the authors also conducted in situ tomographic study of carbonate dissolution. They found that initial structure exerts an important impact on dissolution-induced porosity and permeability evolution. This echoes the understanding that infiltration instabilities amplify small structural differences (heterogeneities) over time. In the paper, the heterogeneity was characterized using the probability density function (PDF) of fluid velocity, an equivalent of residence time distribution (RTD) based on streamline analysis. While effective, this approach also raises the discussion whether the homogeneity in flow distribution necessarily indicate a fairly homogeneous dissolution field - as the streamlines located at the proximity of each other in a PDF could have vastly different access to mineral surface. The impact of flow heterogeneity is discussed in a related study (Pereira Nunes et al., 2016) in a more detailed manner, using three fundamentally different initial structures. By comparing the evolution of flow fields of a beadpack, an oolite limestone and a very heterogeneous limestone, it was demonstrated that the initially most heterogeneous limestone had initially the highest effective reaction rate, as opposed to the homogeneous beadpack and oolite. However, at later stage of dissolution this trend was reversed.

Reactant Distribution and Dissolution Patterns Figure 3a shows an isosurface that separates the developing pore from the rest of the porous medium. The reactive surface area 
(RSA), as defined in Section 2, is part of the geometric surface area (GSA) that serves as the interface for water-rock interaction. It is calculated as the portion of GSA on which the chemical conversion, determined using Equations 3 and 6, is greater than zero. RSA and the rest of GSA (unreactive) are separated by the reaction front. In the flow field, RSA relies heavily on the spreading of the fluid reactivity near the tip of an advancing pore. The slower the fluid reactivity depletes along a streamline, the farther the reaction front extends and the greater the reactive subvolume. Consequently, the apparent solubility of the solid (the total amount of mineral dissolvable in a closed, freely drifting system), the fluid residence time and the geometric surface area available along a flow path determine RSA simultaneously. This quantity ceases to increase as the reaction front extends beyond the fluid outlet and becomes equal to the area of the isosurface for porosity $=0$.

It is worth noticing that fluid breakthrough occurs soon after the extension of reaction front beyond the fluid outlet, marking a significant visual change in dissolution pattern. This pattern switching brings us back to the discussion of defining time zero - i.e. what happens when the initial microstructure already contains a flow path that leaks reactive fluid out? This situation can be identified by tracking the effluent composition. For example, Menke et al. emphasized the impact of initial microstructure on pore development by looking into the correlation between effluent $\mathrm{pH}$ of core flooding and carbonates' batch dissolution rates (Menke et al., 2017). They noticed that even in Ketton, a less heterogeneous medium, different initial pore structures could lead to channelisation at a lower $\mathrm{pH}$ while uniform dissolution at higher. This is an important observation because a lower $\mathrm{pH}$ yields a greater reactant penetration depth and therefore favors a more uniform pattern. In addition, the authors reported results with a significant amount of aqueous $\mathrm{CO}_{2}$. The $\mathrm{pH}$ increased by roughly one unit within the sample dimension during the early stages of core flooding, when there was a better fluid-solid contact. This extent of increase indicates that the fluid was still reactive after leaving the sample. Given that the reaction rate is not very sensitive to $\mathrm{pH}$ variation within this unity regime, the effluent $\mathrm{pH}$ may be used as an indicator for macroscopic carbonate dissolution rate with a slight underestimation.

Pore Growth Controlled by Reaction Front Migration The simulated microscopic pore grows after the migration of the reaction front for two reasons. First, the reaction front encompasses the reactive subvolume of the medium. At any instant, pore can only grow within this defined space. Second, the flow path with the farthest reaching reaction front also dissolves fastest. Two important implications follow: 1) both the reaction front migration and pore growth follow streamlines in a flow field. Along the same streamline, the tip of a pore has the highest reactivity (where fluid starts to contact solid) while the reaction front has the lowest reactivity (as the fluid approaches equilibrium);2) the streamline with the largest initial distance between the pore tip and the reaction front is the axis of pore growth. This is because of spontaneous fluid focusing - the advantage in reactivity along this winning streamline is constantly enhanced over time by the positive coupling between flow and dissolution. Similar streamline based analysis has been reported. For example, Pereira Nunes et al. developed a tracing approach to investigate the reactive transport in heterogeneous rocks (Pereira Nunes et al., 2016). The approach solves Navier-Stokes equations to obtain the flow field, which is then considered the carrier of reactive solutes. The flow streamlines form the basis for the advective transfer of solutes, on top of which is diffusion considered the random motion of solute as particles. This approach demonstrated efficiency in tracking the temporal evolution of dissolution rate variations in space. In addition, this approach holds the potential of differentiating the residence time and the access to solid surfaces for each and every streamline. The latter is a key to the interpretation of complex reactive transport 
phenomena in natural porous materials - especially the various visual "dissolution regimes" categorized by macroscopic dimensionless numbers such as Pe and Da.

\subsection{Identifying the reaction front position}

Defining Cumulative Surface The position of the reaction front can be estimated by considering streamlines as flow paths with infinitesimal cross sections and by defining cumulative surface $(\mathrm{CS})$ as

$$
\mathrm{CS}=\int_{s} S S A \cdot d s / \mathbf{v}
$$

where $s$ indicates integral along a streamline, $S S A\left(\mathrm{~m}^{-1}\right)$ represents specific surface area, (i.e. GSA per unit volume, which is position dependent) and $v$, the velocity. $d s / v$ gives $d \tau$ which is the differential residence time of a fluid element travelling along the integrating path. A probability density function of CS for the complete flow field can then be estimated by applying Equation 14 to a sufficiently large sample of streamlines that originate from the fluid inlet. In the inset of Figure $3 \mathrm{a}$, we show the shape of a probability distribution based on the CS of 40,000 streamlines (one from each inlet voxel) at the beginning of the simulation, when the microstructure was intact. For the convenience of discussion, we assume segregated flow in the following analysis. This assumption means that fluid flowing in different streamlines do not exchange mass (no mixing). It follows that for each streamline,

$$
\int_{0}^{\tau} S S A \cdot d t=\int_{0}^{\left[\mathrm{Ca}^{2+}\right]} \frac{d\left[\mathrm{Ca}^{2+}\right]}{r_{d i s s}(\mathrm{pH}, \mathrm{SI})},
$$

where $\int_{0}^{\tau} S S A \cdot d t$ gives the CS for a specified residence time, $\tau(\mathrm{s} / \mathrm{m}),\left[\mathrm{Ca}^{2+}\right]$ represents the aqueous concentration of calcium ions (M) and $r_{\text {diss }}$ represents the rate of calcite dissolution $\left(\mathrm{mol} \cdot \mathrm{m}^{-2} \cdot \mathrm{s}^{-1}\right)$, which depends on the saturation index $(\mathrm{SI})$ of calcite and the $\mathrm{pH}$. Releasing calcium from the solid increases $\mathrm{pH}$ and SI, both decrease the dissolution rate. According to the RHS of Equation 15, the reaction front extends farther along a flow path with a lower chemical conversion. Combined with Equation 14, we conclude that microscopic pore growth favors the streamline with minimal cumulative surface. Such a streamline has the lowest chemical conversion per unit distance and thus a far reaching reaction front. More importantly, both features are preserved and enhanced over time, by the flow-reaction coupling, as the pore structure and flow field evolve.

The effect of the cumulative surface on pore development is visualized in Figure 3. The morphology of the micropore at the cusp of breakthrough (porosity $=0.44$ ) closely resembles the shape of the streamlines derived from the initial flow field (Fig. 3a). These streamlines are in the lowest decile of cumulative surface (inset, red bars) before the microstructure was modified by the reactive fluid. This resemblance suggests the feasibility of correlating the trajectory of pore growth with CS. In Figure 3b, we show five streamlines in the initial flow field when the overall porosity was 0.20 . For each, we determined the CS using Equation 14 and the dissolution rate using Equation 15 (Fig. 3c). Dissolution rate defines the positions of the reaction front. For example, the grey dashed line cuts the curves at the required travel distance for decreasing the dissolution rate to $5 \mu \mathrm{mol} \cdot \mathrm{m}^{-2} \cdot \mathrm{s}^{-1}$. If this (arbitrarily chosen, as the rate may approach absolute zero asymptotically) value represents that of the reaction front, the yellow streamline would have the shortest reactive length $(\sim 10$ $\mu \mathrm{m})$ while the red one has the longest $(\sim 27 \mu \mathrm{m})$. With the initial microstructure, no fluid induced pore exists and therefore its tip is the fluid inlet (at $s=0 \mu \mathrm{m}$ ). The red streamline is 
expected to become the path of the dominating pore growth because it has the largest Damköhler length, the distance between pore tip and reaction front. If this sieving process is repeated for streamlines representing the complete initial flow field, the axis of a winning pore can be estimated without evolving the microstructure - given that the process is dominated by positive flow-reaction coupling.

Effects of CT Resolution and Grain Relocation There are, however, two issues that ought to be pointed out. First, it is apparent from the simulation that dissolution localization occurs only at scale larger than the voxel. This implies that the macroscopic behavior is independent of intra-voxel variability and that the flow field, mixing as well as solid-fluid contacting are all homogenized within each voxel. As we discussed in the introduction and in Section 2, we argue that there is no such scale separation in reality-dissolution localization occurs wherever a positive feedback between advection and mineral dissolution exists. If the interior structure of a voxel creates a heterogeneous flow field, pores will develop preferentially along those streamlines with lower cumulative surface. The apparent scale separation is a caveat that is imposed by the hard limit of the characterization resolution. It reduces the fidelity of reproducing the reactive transport in the porous sample. However, this homogenization does not change the definition of CS, nor does it affect how CS is calculated. Moreover, the very high resolution of the digital model (25 nm/voxel) serves to minimize the impact of this homogenization.

Another caveat is that the relocation of grains with the stream and the mechanical sustainability of the structure are ignored. In our model, the voxel porosity is allowed to reach unity. This induces a conceptual issue: there is an upper limit of porosity above which a cohesive carbonate matrix cannot be sustained. The microstructure disintegrates, producing solid particles that may be displaced by the fluid or gravity. When the particles accumulate at pore throats, they can exert important control on the flow field, and thus on the dissolution localization. This issue can be circumvented by assuming an inert silicate matrix and thus capping the upper porosity of elemental units in a simulation (Deng \& Peters, 2018; Deng et al., 2018). In a related paper, we discussed this issue in the context of how these mechanical impacts disrupt the positive feedback loop (Yang, Hakim, et al., 2018). However, a quantitative treatment is still beyond our reach. Also, when running the simulation, we did not consider the reactivity difference between the biogenic materials (calcispheres) and the abiotic calcite. Although they are both $\mathrm{CaCO}_{3}$, the association of organic compounds, e.g. coccolith associated polysaccharides, can modify dissolution behavior (Hassenkam et al., 2011).

\subsection{Preferential flow path formation recorded by in situ $\mu \mathrm{CT}$}

Direct Observation of Pore Growth We recorded the development of chalk pore structure during dissolution using in situ X-ray microtomography $(\mu \mathrm{CT})$. The smallest achievable voxel size (i.e. the highest resolution) with the in situ setup was $248 \mathrm{~nm}$. Although with this resolution, the geometric surface area could not be as accurately characterized as with nanoCT, the in situ scans allowed us to track the evolution of the pores and to distinguish the preexisting ones from those developed during dissolution. In Figure 4, we show $200 \square 245 \mu \mathrm{m}^{2}$ cross sections of a time series collected over a 21 hour experiment. The direction of fluid flow is normal to the image plane and points inwards. Perspective 3D views of the microstructure $\left(200 \square 245 \square 300 \mu \mathrm{m}^{3}\right)$ before and after the percolation are presented in Figure 5a and $\mathrm{b}$. The major flow channel advanced gradually in the flow direction and expanded in diameter from the lower right of the region of interest (ROI). The preferential removal of material is demonstrated in the distinct dissolution region within the ROI. Near the lower right are flow paths with shorter residence time and thus lower cumulative surface. 
Fluid in this region is farther from equilibrium and therefore dissolves chalk faster. In contrast, the streamlines going through the porous area on the left side of the ROI are expected to have a greater cumulative surface. The fluid following these paths was therefore closer to saturation and dissolved chalk slowly. A comparison between Figure 4a to j shows that the left side of the ROI did become more porous with time but the fine, grainy texture was preserved (see also Fig. 5b). As time elapsed, more fluid was directed to the right because of the greater permeability. Given a constant macroscopic volumetric flowrate, this flow stratification further increased the residence time in the less porous region, resulting in the positive feedback. Overall, the in situ observation agrees qualitatively with the model simulation. The evolution of the microstructure met the expectation of a cumulative surface based analysis, although the size of the developing flow channel was significantly greater than that in the simulation in light of a higher flow rate $(0.01 \mathrm{~mL} / \mathrm{min})$.

The emergence of clearly identifiable pores from a fairly uniform structure, then followed by pore expansion (Fig. 4), has been reported before. Al-Khulaifi et al. reported two-stage dissolution of carbonates aligned in series (dolomite-limestone) in the flow direction. The first stage was characterized by the graduate emergence of a preferential flow path from the visually uniform limestone, whereas the second stage showed the slow expansion of the path that channelizes the fluid after structural breakthrough (Al-Khulaifi et al., 2017). This result might indicate that the visually different patterns are two stages of the same process. It is therefore of interest to ask if channel development always initiates within a uniform dissolution pattern in a pre-determined manner. Al-Khulaifi et al. also investigated the impact of mass transfer on the effective dissolution rate of dolomite (Al-Khulaifi et al., 2018). They characterized the initial heterogeneities of dolomite cores using the probability density function (PDF) of simulated velocity field and grouped samples accordingly. This effort marks an important methodological progress because keeping the starting microstructure constant is essential but practically very challenging for geologic materials. In the paper the authors confirmed that new flow channels emerged from the initially uniform medium and that the final morphology and number of channels were affected by the macroscopic flowrate. However, the paper also invites the question whether a velocity PDF is the best indicator for homogeneity when it comes to mineral dissolution in flowing fluid. The question stems from the fact that the (in)efficiency of fluid-solid contacting is not reflected in this function and that both uniform and channelized (localized) dissolution yield a single modal PDF of velocity. The use of CS' PDF (Fig. 5) helps differentiate channelized flow from that in a stagnant zone.

Estimating Cumulative Surface using $\mu$ CT Images $\quad$ In Figure 5, the screening procedure discussed in Figure 3 was applied to the microstructure model that was reconstructed from the $\mu \mathrm{CT}$ datasets, to identify the potential pathways for pore growth. Figure 5c shows the streamlines with the lowest $10 \%$ of CS at the beginning of the experiment. The lowest $2 \%$ are colored red. These lines are expected to be preferential dissolution pathways. The screening serves only as an estimate for two reasons. First, the in situ $\mu \mathrm{CT}$ cannot fully resolve the spatial variations in density for fine grain materials such as chalk. The CS is therefore underestimated. Second, the cropped domain covers only a subvolume of the cylindrical sample. The numerically imposed flow field thus does not fully reproduce the distribution of streamlines in the actual sample. Nevertheless, the clustering of the streamlines in the intact microstructure strongly suggests pore development near the lower right of the ROI. In Figure 5d, we drew streamlines originating from the same points as in Figure 5c, but based on an updated flow field. Red color indicates streamlines with the lowest cumulative surface. Fluid in these streamlines bypasses the porous material completely and does not contribute to dissolution. The effect of CS on pore development is 
manifested clearly in the change of the probability distribution, shown in Figure $5 \mathrm{e}$ and $\mathrm{f}$. The original heterogeneity of chalk produces a distribution of CS that spans two orders of magnitude, with a mean of $1.23 \square 10^{7} \mathrm{~s} / \mathrm{m}$ and standard deviation of $8.43 \square 10^{6} \mathrm{~s} / \mathrm{m}$. After channelization, the probability density function shows a typical bimodal distribution, with one mode near zero, representing the bypassing flow, and the other mode at a larger CS. The probability density function in Figure 5 f covers 14 orders of magnitude and yields an average CS of $4.0 \square 10^{10} \mathrm{~s} / \mathrm{m}$ and a standard deviation of $7.25 \square 10^{11} \mathrm{~s} / \mathrm{m}$.

It is worth emphasizing that over the course of 21 hours imaging we did not observe significant multiphase flow characteristics within the fairly small FOV. This single phase behavior may not be assumed in upscaled systems where oil phase or gaseous/supercritical $\mathrm{CO}_{2}$ is present. Reynolds et al. studied the impact of pore scale heterogeneities on $\mathrm{CO}_{2}$-brine drainage and imbibition and concluded that the spatial organization of capillary heterogeneity is of the primary importance and dominates over porosity and permeability heterogeneities in influencing microscopic behaviors when multiphase flow is concerned (Reynolds et al., 2018). What further complicate a multiphase system are the uncertainties in phase continuity. Isolated fluid phases in capillary-controlled heterogeneous media lack direct interaction with the environment. In a numerical scenario, this disconnectivity is reflected in the lack of information exchange between the fluid mainstream and the disconnected clusters, leading to underscribed boundary conditions and therefore underdetermined systems. Nooruddin et al. developed an upscaling approach to relate pore scale wetting phenomena to Darcy-scale flow. They suggested that large-scale oil trapping in oil-wet or mixed-wet media can be sensitive to local capillary pressure variations (Nooruddin \& Blunt, 2018). In such cases, the PDFs in Figure 5e and $\mathrm{f}$ should be computed using more realistic, multiphase flow fields. The streamlines in the aqueous phase are of primary importance as they define the cumulative surface of water-rock interactions.

\section{Conclusions}

We used X-ray tomography to study the development of microscopic pores in chalk and found a correlation between pore growth and the cumulative surface of streamlines. We show that, in a reactive flow field, pore growth, i.e. preferential flow path formation, is subject to three sequential, recursive processes. First, the tip of a growing pore follows the migration of the reaction front along a streamline. Second, the distance between the pore tip and the reaction front is largest when the cumulative surface along the streamline is lowest because of the interplay between reaction rate, residence time and surface area. Third, the positive feedback between flow and mineral dissolution enhances the leading reaction front over time. Consequently the streamline with the smallest cumulative surface, in the initial microstructure, becomes the favored path for pore growth, irrespective of the ever evolving pore morphology and flow field.

Our result has an important implication. A quantitative treatment of microstructure evolution in natural materials can be daunting. If the migration of reactant is accompanied by pore geometry modification, numerical schemes may need to coherently implement both first principles and phenomenological descriptions in the same simulation domain. The correlation between the initial cumulative surface and the final flow path shed lights on the possibility of predicting pore growth without fully simulating microstructure evolution, but at the same time underlies the importance of material characterization. For example, with sufficient resolution, one can use a steady state flow field to look for the flow path with the minimum cumulative surface in the initial microstructure. This method provides an alternative to percolation experiments or numerical simulations with morphing boundary conditions to identify the preferential dissolution pathway. The ability to image the internal microstructure 
nondestructively in 3D vastly improves geometric characterization and from the results of this work, we argue that it also provides the possibility to predict system evolution directly.

An aspect of porous media evolution that is lacking in this study is the effect of pore structure evolution on the mechanical properties. Confining pressure in may also affect microporosity. In this study, both the numerical simulation and the percolation experiment were made under conditions where the exerted stress did not lead to sample deform or collapse, whereas in field conditions, we know that this happens. A dramatic example is the formation of sink holes in karst terrain. Front instability can also be triggered by processes other than chemical reactions, such as in hydraulic fracturing. The validity of the conclusions from this study is thus limited to scenarios where the pore morphology is not affected by the mechanical strength of the material. We also did not consider other processes that could modify local porosity, such as relocation of grains/particles by fluid flow or mineral precipitation. These effects are discussed in a related paper (Yang, Hakim, et al., 2018). Secondary mineral formation often follows upstream dissolution and becomes more prominent downstream, as cumulative surface increases. In contrast to dissolution, precipitation in a pressure driven flow field leads to negative feedback between chemical reaction rate and flow velocity. Overall, the convolution of processes at the pore scale, where first principles meet phenomenological laws, remains to be better understood, even without considering chemical heterogeneities.

\section{Acknowledgments}

We thank F. Saxild for help with the design and construction of the percolation cell, J. U. Hammel for help with data collection at beamline P05 of Petra III at DESY (Deutsches Elektronen-Synchrotron, Germany), P. Alessandra for help with data collection at the TOMCAT beamline at Swiss Light Source (SLS) at Paul Scherrer Institut (PSI, Switzerland), Y. Zheng for help with data collection with the XRadia Versa 410 system at Technical University of Denmark (DTU) for technical support. We thank the Japan Synchrotron Radiation Research Institute for the allotment of beam time on beamline BL20XU of SPring8 (Proposals 2015A1147 \& 2017A1171). We thank the editor Dr. Xavier Sanchez-Vila, the Associate Editor and seven anonymous reviewers for handling the manuscript and for the numerous thought provoking comments and constructive criticisms.

YY is supported by the European Union's Horizon 2020 Research and Innovation Programme under the Marie Sklodowska-Curie Action project OMNICS (Grant Agreement No 653241) and by the Independent Research Fund Denmark under the Council of Technology and Production grant ConFiG (no. 8022-00162B). Additional funding was provided by the Innovation Fund Denmark through the CINEMA project, as well as the Innovation Fund Denmark and Maersk Oil and Gas A/S through the $\mathrm{P}^{3}$ project. Travel support for synchrotron experiments came from the Danish Agency for Science, Technology and Innovation via Danscatt.

\section{Author contributions}

YY designed the research, built the fluid cell and the greyscale model. YY, HOS and SH conducted the percolation experiments. YY, HOS and KU collected the tomography data. SB processed the images and converted the signals into a porosity distribution. SLSS and HOS advised the research. All authors discussed the results and commented on the manuscript. 


\section{Competing interests}

The authors claim no competing interests.

\section{Data and materials availability}

The complete tomographic time series (147 GB) is deposited on erda.dk and can be accessed using the link https://sid.erda.dk/sharelink/hB5xtx954D

\section{References}

Abdoulghafour, H., Luquot, L., \& Gouze, P. (2013). Characterization of the mechanisms controlling the permeability changes of fractured cements flowed through by $\mathrm{co}_{2}$-rich brine. Environmental Science \& Technology, 47(18), 10332-10338. doi:10.1021/es401317c

Al-Khulaifi, Y., Lin, Q., Blunt, M. J., \& Bijeljic, B. (2017). Reaction rates in chemically heterogeneous rock: Coupled impact of structure and flow properties studied by x-ray microtomography. Environmental Science \& Technology, 51(7), 4108-4116. doi:10.1021/acs.est.6b06224

Al-Khulaifi, Y., Lin, Q., Blunt, M. J., \& Bijeljic, B. (2018). Reservoir-condition pore-scale imaging of dolomite reaction with supercritical co2 acidified brine: Effect of pore-structure on reaction rate using velocity distribution analysis. International Journal of Greenhouse Gas Control, 68, 99-111. doi:10.1016/j.ijggc.2017.11.011

Bruns, S., Stipp, S. L. S., \& Sørensen, H. O. (2017a). Looking for the signal: A guide to iterative noise and artefact removal in X-ray tomographic reconstructions of porous geomaterials. Advances in Water Resources, 105, 96-107. doi:10.1016/j.advwatres.2017.04.020

Bruns, S., Stipp, S. L. S., \& Sørensen, H. O. (2017b). Statistical representative elementary volumes of porous media determined using greyscale analysis of 3d tomograms. Advances in Water Resources, 107, 3242. doi:10.1016/j.advwatres.2017.06.002

Budek, A., \& Szymczak, P. (2012). Network models of dissolution of porous media. Physical Review E, 86(5), 056318 .

Chadam, J., Hoff, D., Merino, E., Ortoleva, P., \& Sen, A. (1986). Reactive infiltration instabilities. IMA Journal of Applied Mathematics, 36(3), 207-221. doi:10.1093/imamat/36.3.207

Daccord, G., Lenormand, R., \& Lietard, O. (1993). Chemical dissolution of a porous medium by a reactive fluid-i. Model for the "wormholing” phenomenon. Chemical Engineering Science, 48(1), 169-178.

Daccord, G., Lietard, O., \& Lenormand, R. (1993). Chemical dissolution of a porous medium by a reactive fluid-ii. Convection vs reaction, behavior diagram. Chemical Engineering Science, 48(1), 179-186.

Deng, H., Ellis, B. R., Peters, C. A., Fitts, J. P., Crandall, D., \& Bromhal, G. S. (2013). Modifications of carbonate fracture hydrodynamic properties by $\mathrm{Co}_{2}$-acidified brine flow. Energy \& Fuels, 27(8), 42214231. doi:10.1021/ef302041s

Deng, H., Fitts, J. P., Crandall, D., McIntyre, D., \& Peters, C. A. (2015). Alterations of fractures in carbonate rocks by $\mathrm{CO}_{2}$-acidified brines. Environmental Science \& Technology, 49(16), 10226-10234. doi:10.1021/acs.est.5b01980

Deng, H., \& Peters, C. A. (2018). Reactive transport simulation of fracture channelization and transmissivity evolution. Environmental Engineering Science. doi:10.1089/ees.2018.0244

Deng, H., Steefel, C., Molins, S., \& DePaolo, D. (2018). Fracture evolution in multimineral systems: The role of mineral composition, flow rate, and fracture aperture heterogeneity. ACS Earth and Space Chemistry, 2(2), 112-124. doi:10.1021/acsearthspacechem.7b00130

DePaolo, D. J., \& Cole, D. R. (2013). Geochemistry of geologic carbon sequestration: An overview. Reviews in Mineralogy and Geochemistry, 77(1), 1-14. doi:10.2138/rmg.2013.77.1 
Ellis, B. R., Fitts, J. P., Bromhal, G. S., McIntyre, D. L., Tappero, R., \& Peters, C. A. (2013). Dissolution-driven permeability reduction of a fractured carbonate caprock. Environmental Engineering Science, 30(4), 187-193.

Fabricius, I. L. (2007). Chalk: Composition, diagenesis and physical properties. Geological Society of Denmark. Bulletin, 55, 97-128.

Fitts, J. P., \& Peters, C. A. (2013). Caprock fracture dissolution and $\mathrm{co}_{2}$ leakage. Reviews in Mineralogy and Geochemistry, 77(1), 459-479. doi:10.2138/rmg.2013.77.13

Fogler, H. S. (2016). Conversion and reactor sizing. In H. S. Fogler (Ed.), Elements of chemical reaction engineering (pp. 33-67): Pearson Education.

Fredd, C., \& Fogler, H. (1999). Optimum conditions for wormhole formation in carbonate porous media: Influence of transport and reaction. SPE Journal, 4(03), 196-205.

Fredd, C. N., \& Fogler, H. S. (1998). Influence of transport and reaction on wormhole formation in porous media. AIChE Journal, 44(9), 1933-1949. doi:10.1002/aic.690440902

Gürsoy, D., De Carlo, F., Xiao, X. \& Jacobsen, C. (2014). Tomopy: a framework for the analysis of synchrotron tomographic data. Journal of Synchrotron Radiation, 21(5):1188-1193.

Hassenkam, T., Johnsson, A., Bechgaard, K., \& Stipp, S. L. S. (2011). Tracking single coccolith dissolution with picogram resolution and implications for $\mathrm{CO}_{2}$ sequestration and ocean acidification. Proceedings of the National Academy of Sciences, 108(21), 8571-8576.

Hinch, E., \& Bhatt, B. (1990). Stability of an acid front moving through porous rock. Journal of Fluid Mechanics, 212, 279-288.

Hoefner, M., \& Fogler, H. S. (1988). Pore evolution and channel formation during flow and reaction in porous media. AIChE Journal, 34(1), 45-54.

Holger, O., \& Sjaam, O. (2015). Wormhole formation and compact dissolution in single- and two- phase $\mathrm{co}_{2}{ }^{-}$ brine injections. Geophysical Research Letters, 42(7), 2270-2276. doi:10.1002/2015GL063582

Landrot, G., Ajo-Franklin, J. B., Yang, L., Cabrini, S., \& Steefel, C. I. (2012). Measurement of accessible reactive surface area in a sandstone, with application to co 2 mineralization. Chemical Geology, 318, $113-125$

Li, L., Peters, C. A., \& Celia, M. A. (2006). Upscaling geochemical reaction rates using pore-scale network

modeling. Advances in Water Resources, 29(9), 1351-1370.

Lin, Q., Al-Khulaifi, Y., Blunt, M. J., \& Bijeljic, B. (2016). Quantification of sub-resolution porosity in carbonate rocks by applying high-salinity contrast brine using $\mathrm{X}$-ray microtomography differential imaging. Advances in Water Resources, 96, 306-322. doi:10.1016/j.advwatres.2016.08.002

Luquot, L., \& Gouze, P. (2009). Experimental determination of porosity and permeability changes induced by injection of $\mathrm{CO}_{2}$ into carbonate rocks. Chemical Geology, 265(1-2), 148-159. doi:10.1016/j.chemgeo.2009.03.028

Maher, K., Steefel, C. I., DePaolo, D. J., \& Viani, B. E. (2006). The mineral dissolution rate conundrum: Insights from reactive transport modeling of $\mathrm{u}$ isotopes and pore fluid chemistry in marine sediments. Geochimica et Cosmochimica Acta, 70(2), 337-363.

Menke, H. P., Andrew, M. G., Blunt, M. J., \& Bijeljic, B. (2016). Reservoir condition imaging of reactive transport in heterogeneous carbonates using fast synchrotron tomography - effect of initial pore structure and flow conditions. Chemical Geology, 428, 15-26. doi:10.1016/j.chemgeo.2016.02.030

Menke, H. P., Bijeljic, B., \& Blunt, M. J. (2017). Dynamic reservoir-condition microtomography of reactive transport in complex carbonates: Effect of initial pore structure and initial brine ph. Geochimica et Cosmochimica Acta, 204, 267-285. doi:10.1016/j.gca.2017.01.053

Menke, H. P., Reynolds, C. A., Andrew, M. G., Pereira Nunes, J. P., Bijeljic, B., \& Blunt, M. J. (2018). 4d multi-scale imaging of reactive flow in carbonates: Assessing the impact of heterogeneity on dissolution regimes using streamlines at multiple length scales. Chemical Geology, 481, 27-37. doi:10.1016/j.chemgeo.2018.01.016 
Molins, S., Trebotich, D., Steefel, C. I., \& Shen, C. (2012). An investigation of the effect of pore scale flow on average geochemical reaction rates using direct numerical simulation. Water Resources Research, $48(3)$.

Müter, D., Sørensen, H. O., Jha, D., Harti, R., Dalby, K. N., Suhonen, H., et al. (2014). Resolution dependence of petrophysical parameters derived from x-ray tomography of chalk. Applied Physics Letters, 105(4), 043108. doi:10.1063/1.4891965

Noiriel, C. (2015). Resolving time-dependent evolution of pore-scale structure, permeability and reactivity using x-ray microtomography. Reviews in Mineralogy and Geochemistry, 80(1), 247-285. doi:10.2138/rmg.2015.80.08

Noiriel, C., Steefel, C. I., Yang, L., \& Ajo-Franklin, J. (2012). Upscaling calcium carbonate precipitation rates from pore to continuum scale. Chemical Geology, 318-319, 60-74. doi:10.1016/j.chemgeo.2012.05.014

Nooruddin, H. A., \& Blunt, M. J. (2018). Large-scale invasion percolation with trapping for upscaling capillarycontrolled darcy-scale flow. Transport in Porous Media, 121(2), 479-506. doi:10.1007/s11242-0170960-7

Ortoleva, P., Chadam, J., Merino, E., \& Sen, A. (1987). Geochemical self-organization ii: The reactiveinfiltration instability. American Journal of Science, 287, 1008-1040.

Ortoleva, P. J. (1994). Geochemical self-organization: Oxford University Press: Clarendon Press.

Parkhurst, D. L., \& Appelo, C. (1999). User's guide to phreeqc (version 2): A computer program for speciation, batch-reaction, one-dimensional transport, and inverse geochemical calculations.

Pereira Nunes, J. P., Bijeljic, B., \& Blunt, M. J. (2016). Pore- space structure and average dissolution rates: A simulation study. Water Resources Research, 52(9), 7198-7212. doi:doi:10.1002/2016WR019313

Pokrovsky, O. S., Golubev, S. V., \& Schott, J. (2005). Dissolution kinetics of calcite, dolomite and magnesite at $25 \mathrm{c}$ and 0 to $50 \mathrm{~atm} \mathrm{pco}_{2}$. Chemical Geology, 217(3), 239-255.

Pokrovsky, O. S., Golubev, S. V., Schott, J., \& Castillo, A. (2009). Calcite, dolomite and magnesite dissolution kinetics in aqueous solutions at acid to circumneutral ph, 25 to $150 \mathrm{c}$ and 1 to $55 \mathrm{~atm} \mathrm{pco}_{2}$ : $\mathrm{New}^{-}$ constraints on $\mathrm{co}_{2}$ sequestration in sedimentary basins. Chemical Geology, 265(1), 20-32.

Raeini, A. Q., Bijeljic, B., \& Blunt, M. J. (2017). Generalized network modeling: Network extraction as a coarse-scale discretization of the void space of porous media. Physical Review E, 96(1), 013312.

Raeini, A. Q., Bijeljic, B., \& Blunt, M. J. (2018). Generalized network modeling of capillary-dominated twophase flow. Physical Review E, 97(2), 023308.

Rege, S. D., \& Fogler, H. S. (1989). Competition among flow, dissolution, and precipitation in porous media. AIChE Journal, 35(7), 1177-1185.

Reynolds, C. A., Blunt, M. J., \& Krevor, S. (2018). Multiphase flow characteristics of heterogeneous rocks from $\mathrm{CO}_{2}$ storage reservoirs in the united kingdom. Water Resources Research, 54(2), 729-745. doi:10.1002/2017WR021651

Soulaine, C., Roman, S., Kovscek, A., \& Tchelepi, H. A. (2017). Mineral dissolution and wormholing from a pore-scale perspective. Journal of Fluid Mechanics, 827, 457-483. doi:10.1017/jfm.2017.499

Steefel, C. I., Beckingham, L. E., \& Landrot, G. (2015). Micro-Continuum Approaches for Modeling Pore-Scale Geochemical Processes. Reviews in Mineralogy and Geochemistry, 80(1), 217-246. doi:10.2138/rmg.2015.80.07

Szymczak, P., \& Ladd, A. (2009). Wormhole formation in dissolving fractures. Journal of Geophysical Research: Solid Earth (1978-2012), 114(B6).

Szymczak, P., \& Ladd, A. J. (2014). Reactive-infiltration instabilities in rocks. Part 2. Dissolution of a porous matrix. Journal of Fluid Mechanics, 738, 591-630.

Szymczak, P., \& Ladd, A. J. C. (2012). Reactive-infiltration instabilities in rocks. Fracture dissolution. Journal of Fluid Mechanics, 702, 239-264. doi:10.1017/jfm.2012.174 
Uesugi, K., Hoshino, M., Takeuchi, A., Suzuki, Y., \& Yagi, N. (2012). Development of fast and high throughput tomography using cmos image detector at spring-8. Paper presented at the Proc Soc Photo Opt Instrum Eng.

Wen, H., \& Li, L. (2018). An upscaled rate law for mineral dissolution in heterogeneous media: The role of time and length scales. Geochimica et Cosmochimica Acta, 235, 1-20. doi:10.1016/j.gca.2018.04.024

Yang, Y., Bruns, S., Rogowska, M., Hakim, S. S., Hammel, J. U., Stipp, S. L. S., \& Sørensen, H. O. (2018). Retraction of dissolution front in natural porous media. Scientific Reports, 8, 5693. doi:10.1038/s41598-018-23823-3

Yang, Y., Bruns, S., Stipp, S. L. S., \& Sørensen, H. O. (2017). Dissolved $\mathrm{co}_{2}$ increases breakthrough porosity in natural porous materials. Environmental Science \& Technology, 51(14), 7982-7991. doi:10.1021/acs.est.7b02157

Yang, Y., Bruns, S., Stipp, S. L. S., \& Sørensen, H. O. (2018a). Impact of microstructure evolution on the difference between geometric and reactive surface areas in natural chalk. Advances in Water Resources, 115, 151-159. doi:10.1016/j.advwatres.2018.03.005

Yang, Y., Bruns, S., Stipp, S. L. S., \& Sørensen, H. O. (2018b). Patterns of entropy production in dissolving natural porous media with flowing fluid. PLOS ONE, 13(9), e0204165. doi:10.1371/journal.pone.0204165

Yang, Y., Hakim, S. S., Bruns, S., Rogowska, M., Boehnert, S., Hammel, J. U., et al. (2018). Direct observation of coupled geochemical and geomechanical impacts on chalk microstructure evolution under elevated $\mathrm{co}_{2}$ pressure. ACS Earth and Space Chemistry, 2(6), 618-633. doi:10.1021/acsearthspacechem.8b00013

Yeong, C. L. Y., \& Torquato, S. (1998). Reconstructing random media. Physical Review E, 57(1), 495-506.

Zhang, S., \& Liu, H.-H. (2016). Porosity-permeability relationships in modeling salt precipitation during $\mathrm{Co}_{2}$ sequestration: Review of conceptual models and implementation in numerical simulations. International Journal of Greenhouse Gas Control, 52, 24-31. doi:10.1016/j.ijggc.2016.06.013

Zhao, C., Hobbs, B., \& Ord, A. (2015). Theoretical analyses of chemical dissolution- front instability in fluidsaturated porous media under non- isothermal conditions. International journal for numerical and analytical methods in geomechanics, 39(8), 799-820.

Zhao, C., Hobbs, B. E., \& Ord, A. (2012). Effects of medium and pore- fluid compressibility on chemicaldissolution front instability in fluid- saturated porous media. International Journal for Numerical and Analytical Methods in Geomechanics, 36(8), 1077-1100.

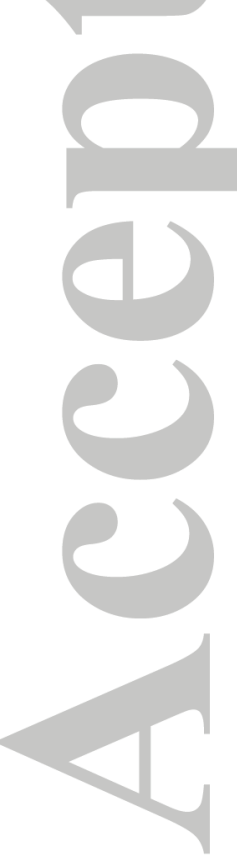


Table 1. List of symbols for the greyscale model. Numbers are given when a single value is used for all reactors. The absence of unit indicates that the quantity is either scaled to a reference value or dimensionless. The dimensionless groups are calculated assuming a time step of 60 seconds. In the actual simulation the time step was adaptive.

\begin{tabular}{|c|c|c|c|}
\hline Symbol & Explanation & Units & Value \\
\hline$|\Delta \varphi|$ & Porosity difference between two voxels connected by a PFR & & \\
\hline$|\Delta \varphi|_{i}$ & Porosity difference between a voxel and its $i$ th neighbor & & \\
\hline & $\begin{array}{l}\text { A lumping coefficient reflecting the dependence of permeability on } \\
\text { voxel porosity }\end{array}$ & $\mathrm{m}^{2}$ & $10^{-13}$ \\
\hline C & Dimensionless $\mathrm{Ca}$ concentration at reactor outlet & & \\
\hline$C_{0}$ & Dimensionless Ca concentration at reactor inlet & & \\
\hline$C_{0, i}$ & Dimensionless Ca concentration in the $i$ th inlet of an MFR & & \\
\hline$C_{A, e q}$ & Equilibrium concentration of $\mathrm{Ca}$ & $\mathrm{mol} / \mathrm{m}^{3}$ & 0.0084 \\
\hline$C_{A, i n j}$ & Injection concentration of $\mathrm{Ca}$ & $\mathrm{mol} / \mathrm{m}^{3}$ & 0 \\
\hline$C_{A 0}$ & Ca concentration at reactor inlet & $\mathrm{mol} / \mathrm{m}^{3}$ & \\
\hline & Damköhler number (Eqs 4 \& 7) & & \\
\hline DS & Dimensionless number (Eq. 9) & & 0.0074 \\
\hline$d t$ & Time step & $\mathrm{s}$ & 60 \\
\hline & $\begin{array}{l}\text { Voxel level mixing factor which ranges from } 1 \text { (complete) to } 0 \text { (no) } \\
\text { mixing. }\end{array}$ & & 0.5 \\
\hline$H n$ & Dimensionless number (Eq. 5) & & 332.5 \\
\hline & Porosity & & \\
\hline & Dimensionless first order rate constant & & \\
\hline$k_{A}{ }^{0}$ & First order rate constant at the injection concentration of $\mathrm{Ca}$ & $\mathrm{m} / \mathrm{s}$ & 0.0033 \\
\hline K & Dimensionless linearized rate constant & & \\
\hline & Linearized rate constant & $\mathrm{m} / \mathrm{s}$ & \\
\hline & Voxel dimension & $\mathrm{nm}$ & 25 \\
\hline$l_{n}$ & Normalized voxel dimension & & 1 \\
\hline$L_{\text {ref }}$ & Characteristic length & $\mathrm{m}$ & $2.5 \square 10^{-8}$ \\
\hline$\mu$ & Fluid viscosity & Pa.s & $8.9 \square 10^{-4}$ \\
\hline$M$ & Molar density & $\mathrm{kg} / \mathrm{mol}$ & 0.1 \\
\hline$p$ & Dimensionless pressure & & \\
\hline$P_{\text {ref }}$ & Reference pressure & $\mathrm{Pa}$ & 1 \\
\hline & Dimensionless volumetric flow rate & & \\
\hline$q_{i}$ & Dimensionless volumetric flow rate from the $i$ th inlet of an MFR & & \\
\hline$Q_{\text {ref }}$ & Reference volumetric flow rate (per inlet voxel) & $\mathrm{m}^{3} / \mathrm{s}$ & $6.25 \square 10^{-21}$ \\
\hline$\rho$ & Density & $\mathrm{kg} / \mathrm{m}^{3}$ & 2710 \\
\hline$r_{A}$ & Calcite dissolution rate & $\mathrm{mol} / \mathrm{m}^{2} / \mathrm{s}$ & \\
\hline$R z$ & Dimensionless number (Eq. 2) & & 449.4 \\
\hline
\end{tabular}



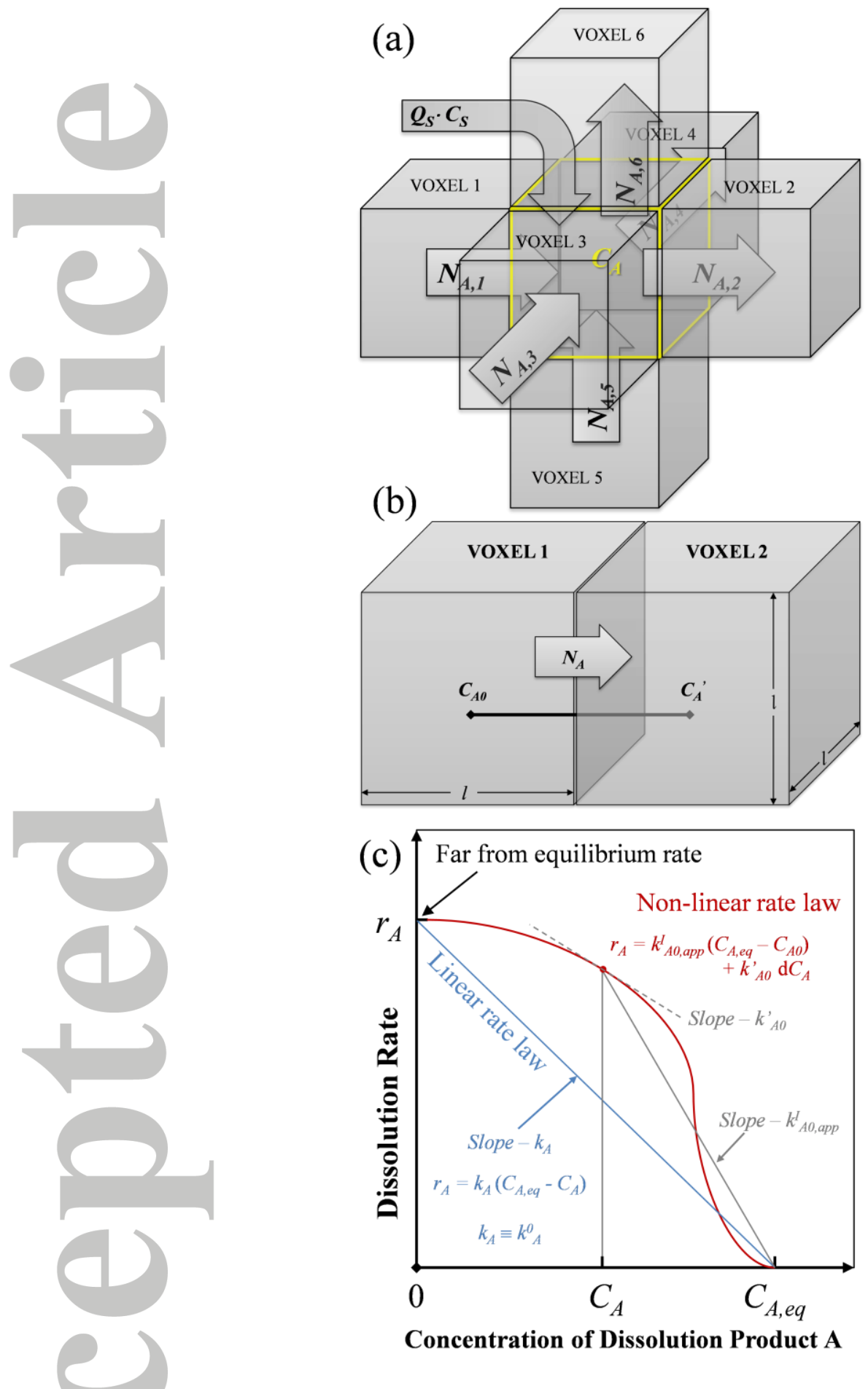

Figure 1. A reactor network model based on greyscale tomography. (a) Each voxel is modelled as a mixed flow reactor (MFR). Reactor porosity was estimated from X-ray intensity using a multi-Gaussian model. The surface area is calculated as the summation of porosity differences between the voxel and its six neighbors. (b) The connection between two voxels is modelled as a plug flow reactor (PFR). The pressure drop occurs only in PFRs. (c) Illustration of the various rate constants $(k)$ used in the model. See also Section 2.2 and Table 1. 

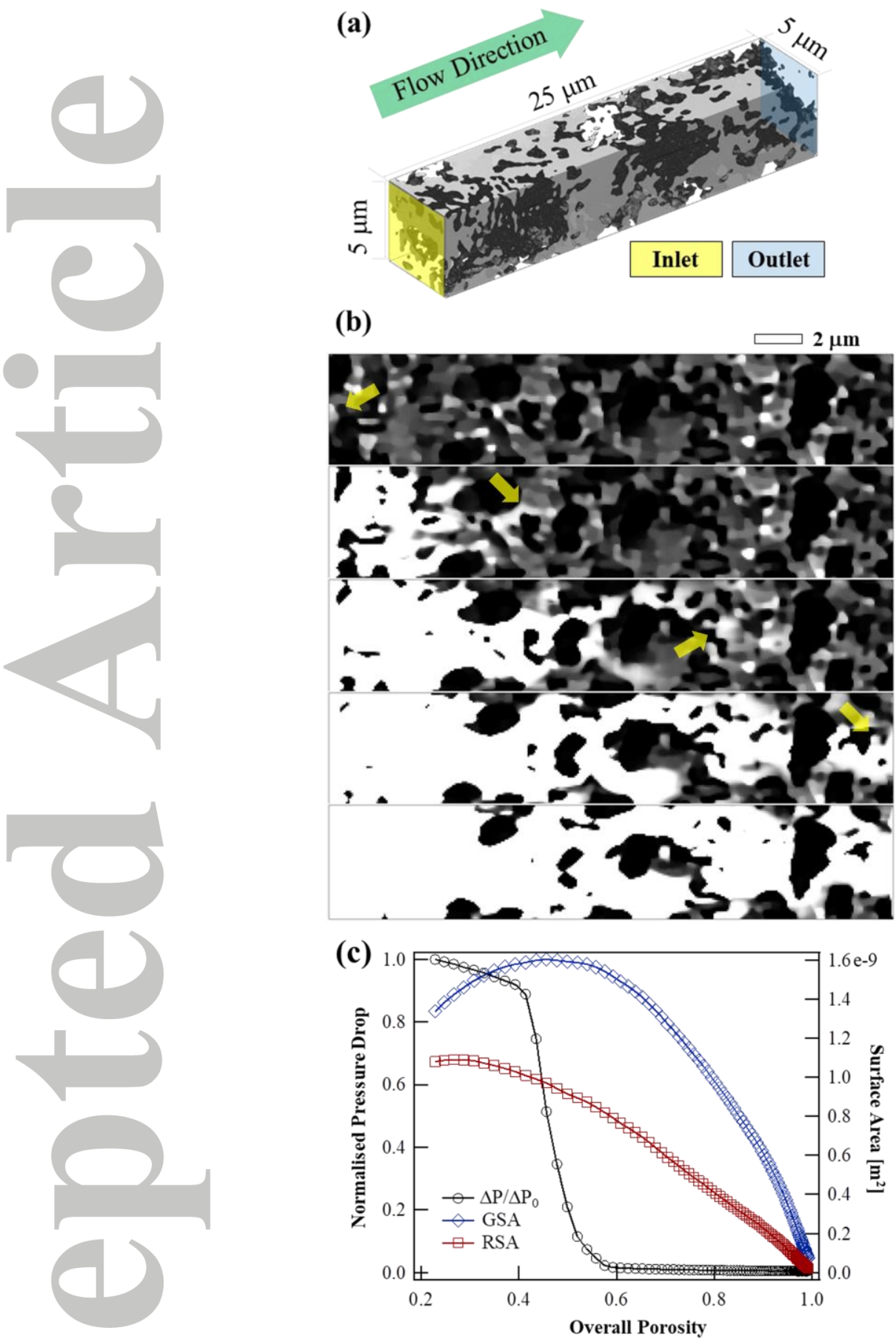

Figure 2. Simulated pore growth in chalk. (a) The domain consists of 40 million voxels representing a $5 \square 5 \square 25 \mu^{3}$ chalk cuboid. The isosurface is drawn at $20 \%$ porosity. (b) Cross sections $\left(5 \square 25 \mu^{2}\right)$ at 4 selected time steps, where the overall porosities of the volume are $0.2,0.3,0.4$ and 0.5 (top to bottom). Pixel greyvalue indicates porosity and is scaled between 1 (void space, white) and 0 (pure solid, black). Arrows point to the advancing pore tips. (c) Evolution of the pressure drop across the sample in the flow direction (normalized to initial value, $\Delta \mathrm{P} / \Delta \mathrm{P}_{0}$, left axis), geometric surface area (GSA, right axis) and reactive surface area (RSA, right axis). 

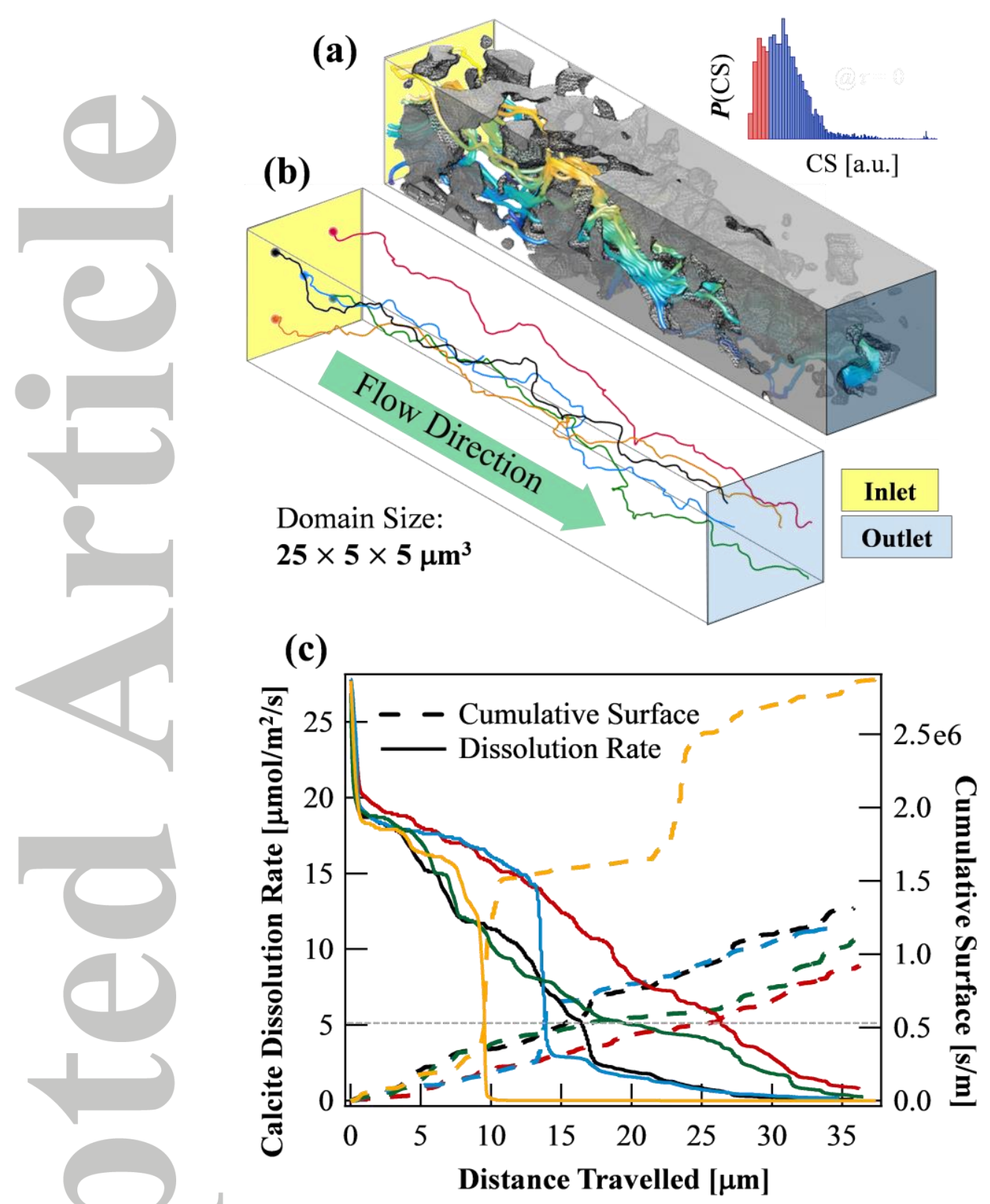

Figure 3. Impact of cumulative surface on pore growth. (a) The microstructure at the cusp of breakthrough (porosity $=0.44$ ). Imposed are the streamlines that have been calculated from the initial flow field (porosity $=0.20$ ). Although the flow field and the microstructure were evolving, these streamlines with far-reaching reaction fronts remained fairly stagnant. The isosurface is drawn at porosity $=1$. The inset shows the shape of the probability density function (PDF) of cumulative surface, calculated from 40,000 streamlines originating from the fluid inlet. The streamlines within the lowest $10 \%$ of CS are red. (b) Five streamline examples. (c) The rate of calcite dissolution and the cumulative surface as functions of travelling distance. Color code corresponds to (b). The streamlines have similar length and tortuosity, but differ greatly in cumulative surface and thus rate profile. The grey dashed line cuts the curves at the position where the dissolution rate in the streamlines drops to 5 $\mu \mathrm{mol} \cdot \mathrm{m}^{-2} \cdot \mathrm{s}^{-1}$. 


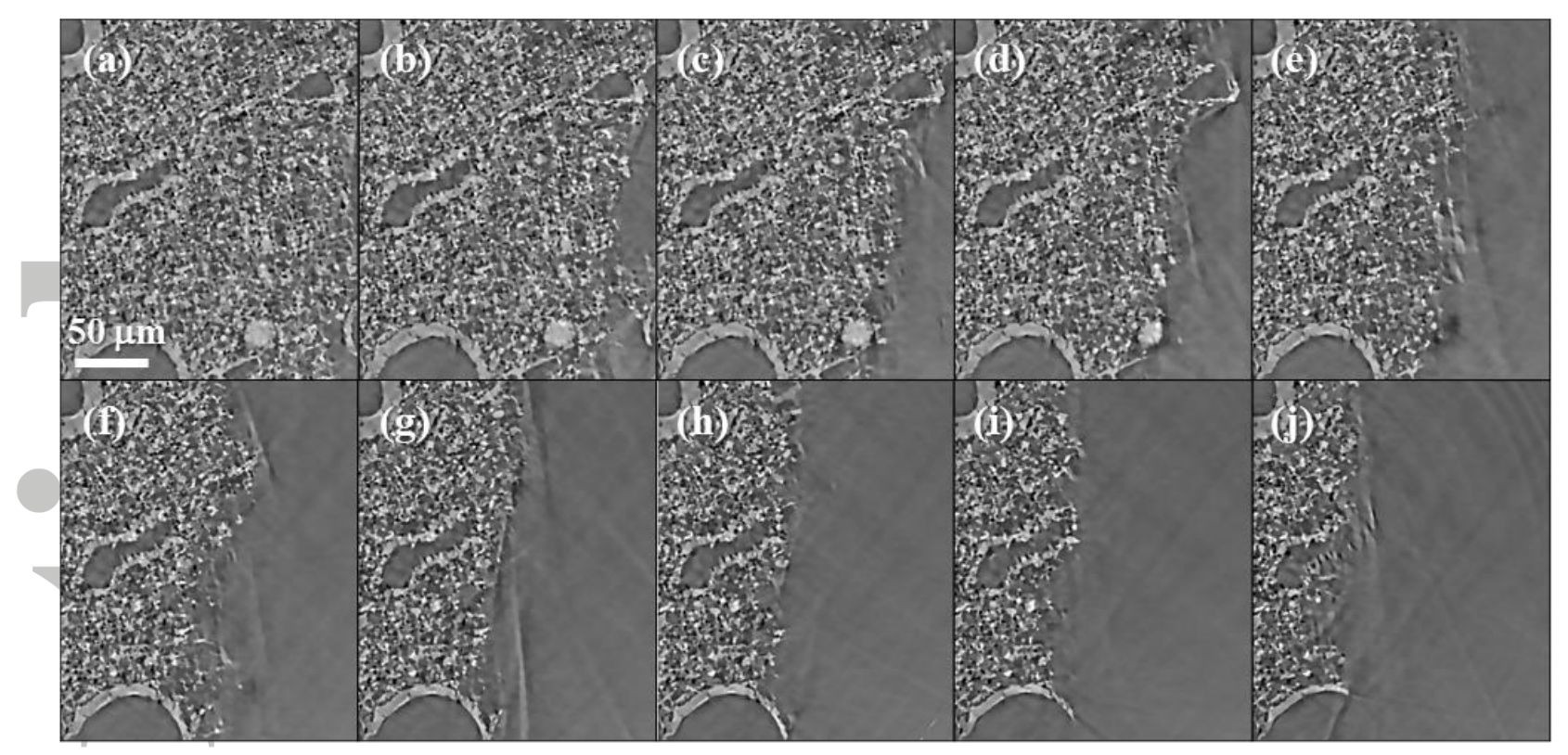

Figure 4. Flow channel formation in chalk. (a) - (j) snapshots from a in situ X-ray tomography timeseries over 21 hours: cross section of a $200 \square 245 \square 300 \mu \mathrm{m}^{3}$ dissolving chalk sample (3D view in Fig. 5). Here, the flow is normal to the plane of display, pointing into the page, away from the reader. A pore originated from the lower right and expanded left- and upwards, while advancing along the flow direction. Solid on flow paths with small cumulative surface (near the pore) was removed preferentially, whereas the grain textures on the left was essentially intact.

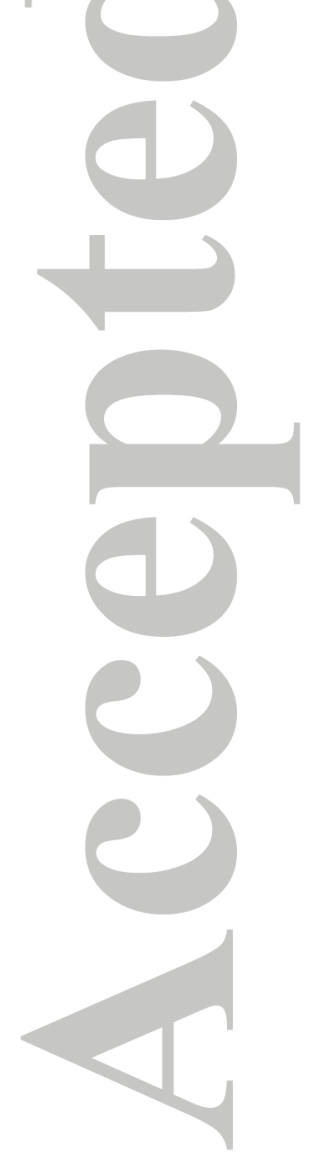




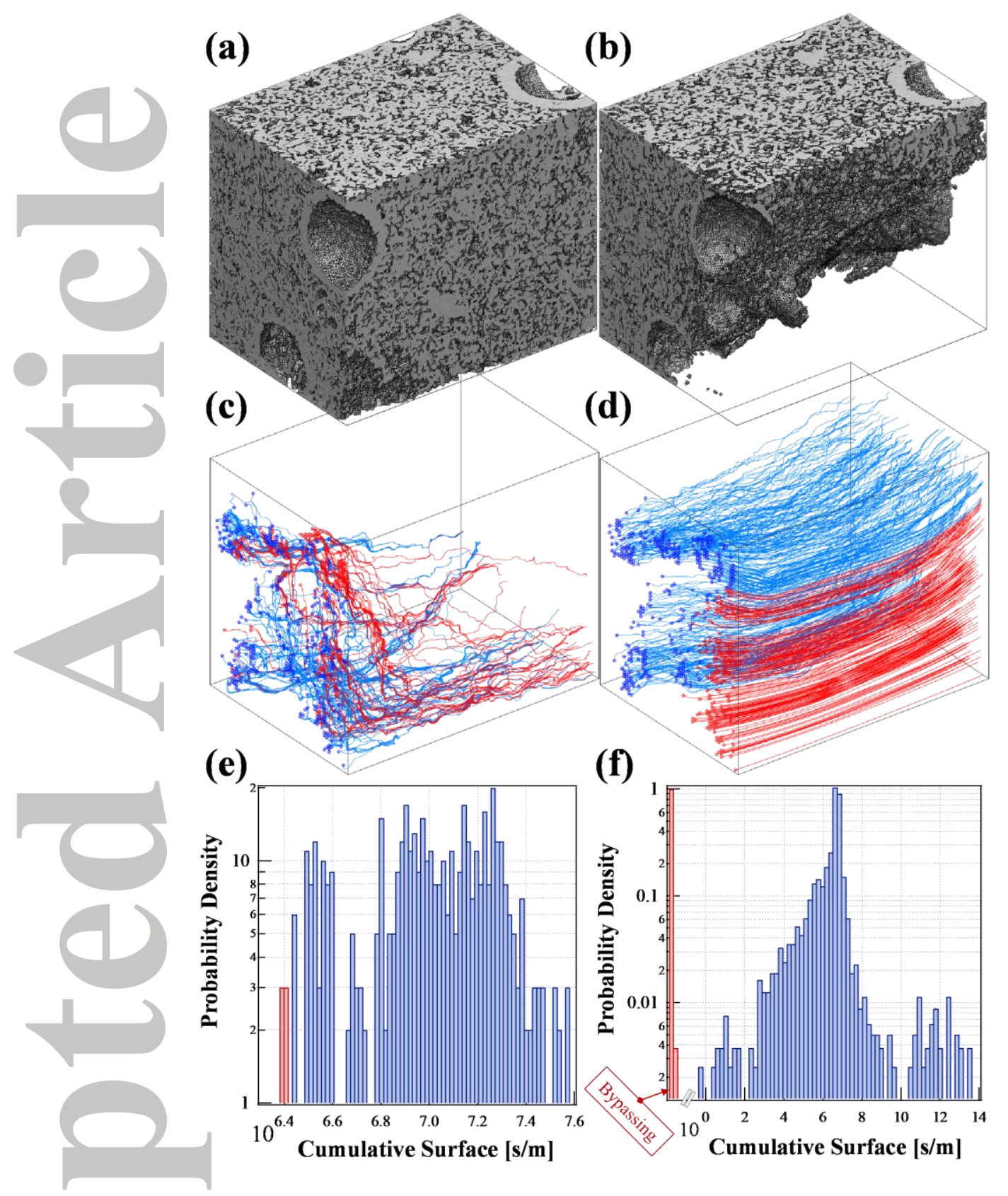

Figure 5. Effect of cumulative surface on flow channel development. (a) and (b) are the 3D images of the subvolume (200 $\square 245 \square 300 \mu^{3}$ ) before and after 21 hours of dissolution in flowing carbonated water. (c) Streamlines with the lowest $10 \%$ of the cumulative surface, among which the lowest $2 \%$ are colored red. The streamlines are determined from the flow field in the initial microstructure. (d) Streamlines originating from the same points depicted in (c) based on the flow field in the final microstructure. (e) and (f) Distribution of cumulative surface before and after the experiment. The long tails of both distributions, which contribute $\sim 10 \%$ of the total probability towards greater cumulative surface, are not shown. The PDF are normalized to the logarithm of cumulative surface so the integral area is 1. The red bars correspond to the red streamlines in (c) and (d). In the $x$ axis of (f), there is a gap below 1 because the bypassing fluid elements have cumulative surface values close or equal to zero. 